DOI 10.32370/2018_09_8

\title{
Establishing Patterns of Formation and Distribution of Primes to Infinity (Riemann Hypothesis)
}

\author{
Rustem Mustafaev
}

\begin{abstract}
The work analyzed the formation of prime numbers in connection with complex odd numbers, a formula for determining primes to infinity was established.

Prime numbers, the location of which they tried to explain with the help of the asymptotic distribution law of primes, are important in cryptography, navigation, and simulation, that is, their definition is of practical importance.

Keywords: Pr (prime); S - (complex number); A - group; S "5"; Z - group; D - group; forming residue; absolute vector I $\vec{a} \mathrm{I}$; the foundation; - ; root; square root; in function Pr; subgroup S "33"; set of X; square odd; $\mathrm{n}$ orders; Pr function "13"; complex squares; increment $(\Delta \mathrm{n})$; Q - values; $\Delta \mathrm{n}=\mathrm{n}_{2}-\mathrm{n}_{1} ; \mathrm{n}$ - even degree; combining number; $\mathrm{S}[\mathrm{L}]$; intermediate complex; identification; increments of squares; interval; from "81"; the amount of increments; exclude; $\{\mathrm{P} \mathrm{r} \rightarrow \infty$.
\end{abstract}

Гипотеза Римана о распределении нулей дзета - функции Римана была сформулирована Бернхардом Риманом в 1859 году.

В то время, как никто не видел какой - либо закономерности, описывающей распределение простых чисел, Риман обнаружил некоторую закономерность.... «Основным свойством простых чисел есть невозможность деления на любое иное число ( кроме 1) без остатка. Простое число, назовем Pr, не образуется функцией умножения разных чисел (нечетных), возведением нечетных чисел в степень. Например, 3; 5; 7; 11; 13; 17; 19; 23; 29, и далее, до бесконечности ( $\{\mathrm{P} \mathrm{r} \rightarrow \infty-$ множество простых чисел стремится к бесконечности), - невозможно разделить никакое число так, чтобы получить целое число (кроме себя и «1»).

Это $1 ; 3 ; 5 ; 7 ; 11 ; 13 ; 17 ; 19 ; 23 ; 29 ; 31 ; 37 ; 41 ; 43 ; 47 ; 53 ; 59 ; 61 ; 67 ; 71 ; 73 ; 79 ; 83 ; 89$; $97 ; 101 ; 103 ; 107 ; 109$ и далее до бесконечности.

Значения $15 ; 25 ; 35 ; 45 ; 55 ; 65 ; 75 ; 85 ; 95 ; 105 ; 115$, и далее все, оканчивающиеся на «5» и образованные суммой «Х»- пятизначений, увеличением «5» в n- количество раз, являются цельноделимыми на «5», и являются сложными нечетными числами... 15:5=3; 25:5=5; 35:5=7; 45:5=9; 55:5=11; 65:5=13; 75:5=15; 85:5=17..и далее до $\infty$ по условной формуле: $\mathrm{A}=5 \mathrm{xn}, \mathrm{n}$ - порядковый номер числа сложных $\mathbf{A}$ группы.

Примечание: в формулах: $\mathrm{A}=5 \mathrm{xn} ; \mathrm{A}=5+10 \mathrm{xn}$ и подобных, символ «х» обозначает умножение. 
Также эти числа можно определить в закономерности: $\mathbf{A = 5 + 1 0 x n , ~ г д е ~ « 5 » ~ я в л я е т с я ~}$ точкой отсчета, при $\mathrm{n}=0 \ldots$

A - группу обозначим $\mathbf{S}$ « 5 », $\mathbf{S}$ - сложные числа. Значит, все простые числа $\mathrm{Pr}$, в конце могут иметь (оканчиваться) значения $1 ; 3 ; 7 ; 9 \ldots$

Первым числом в ряду $\operatorname{Pr}$ есть «1»; первым нечетным числом, дающим при делении результат, отличный от числителя, есть «3».

Рассмотрим деление начальных простых чисел на «3».. 5:3=1,6666.. 7:3=2,6666...; $11: 3=3,6666 \ldots ; 13: 3=4.3333 \ldots ; 17: 3=5.6666 \ldots ; 19: 3=6.3333 \ldots ; 23: 3=7.6666 \ldots$; $29: 3=9,6666 \ldots ; 31: 3=10,3333 \ldots ; 37: 3=12.3333 \ldots ; 41: 3=13,6666 ; 43: 3=14,3333 \ldots$; $47: 3=17,6666 \ldots$ Всегда при делении на 3 образуется остаток, 0,3333... или 0,6666...

Узнаем значения чисел при делении которых на «8»; «18»; «28»; «38», любые четные числа с окончанием на «8» и остатке 0,3333 и $0,6666 \ldots$ - образуется значение «3».

$\mathrm{X}: 8,3333 \ldots=3 ; \mathrm{X}=24,999998 \approx 25 ; \mathrm{X}: 8,6666 \ldots=3 ; \mathrm{X} \approx 26 ; \mathrm{X}: 18,3333 \ldots=3 ; \mathrm{X} \approx 55$; $\mathrm{X}: 18,6666 \ldots=3 ; \mathrm{X} \approx 56 ; \mathrm{X}: 28,3333 \ldots=3 ; \mathrm{X} \approx 85 ; \mathrm{X}: 28,666 \ldots=3 ; \mathrm{X} \approx 86 \ldots$ в данных

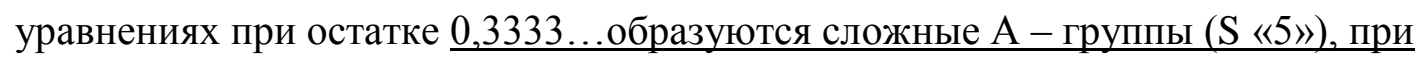
остатке $0,6666 \ldots$ - четные числа. Значение остатка влияет на образование числа, остаток является «образующим», и обозначается как «І $\overrightarrow{\mathrm{I}}$ » (абсолютный $\alpha$ вектор»)... Все простые числа должны содержать определенное количество минимальных простых чисел и остаток как целое число, отличное от этих минимальных значений (назовем его «Х»).

Эти минимальные простые значения являются основой простых чисел, их корнями. Если корень обозначить как - , то $\operatorname{Pr}=-\mathbf{x} \mathbf{n}+\mathbf{X}$, где $\mathbf{n}-$ количество корней, $\mathbf{X}-$ остаток.

Множество простых чисел образуется способом: $\operatorname{Pr}=3 \mathrm{xn}+\mathrm{X} ; \operatorname{Pr}=5 \mathrm{xn}+\mathrm{X} ; \mathrm{Pr}=7 \mathrm{xn}+\mathrm{X}$; $\operatorname{Pr}=11 \mathrm{xn}+\mathrm{X} ; \operatorname{Pr}=13 \mathrm{xn}+\mathrm{X} ; \operatorname{Pr}=17 \mathrm{xn}+\mathrm{X} ; \mathrm{Pr}=19 \mathrm{xn}+\mathrm{X} ; \ldots$ и далее, аналогично, до бесконечности... Возведение в квадратную (вторую) степень (Y= $\operatorname{Pr}$ х $\operatorname{Pr}$ ) любого простого числа (кроме»5») образует нечетное значение с окончанием на «1» или «9»: $11^{2}=121 ; 13^{2}=169 ; 17^{2}=289 ; 19^{2}=361 ; 23^{2}=529 ; 29^{2}=841 ; 31^{2}=961 ; 37^{2}=1369 \ldots$

Рассмотрим деление на «3» небольших значений квадратов простых чисел.

$121: 3=40,3333 \ldots ; 169: 3=56,3333 \ldots ; 289: 3=96,3333 \ldots ; 361: 3=120,3333 \ldots$;

$529: 3=176,3333 \ldots ; 841: 3=440,3333 \ldots ; 961: 3=320,3333 \ldots ; 1369: 3=456,3333 \ldots$

Если начальные значения квадратов делятся на «3» с образованием четных чисел с остатком «I $\overrightarrow{\boldsymbol{a}} \mathbf{I} »=0,3333 \ldots$, тогда верно, что множество простых чисел можно образовать произведением «3» на четные числа в сумме с остатком 0,3333 ... ( \{ 


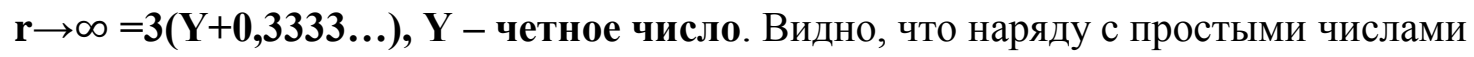
образуются сложные при некоторых значениях «Ү». При Y=16; 40; 56; 96; 120; 176,образуются сложные, как квадраты простых чисел: 49; 121; 169; 289; 361; 529 и другие $: \sqrt{49}=7 ; \sqrt{121}=11 ; \sqrt{169}=13 ; \sqrt{289}=17 ; \sqrt{361}=19 ; \sqrt{529}=23$.

Вывод: для исключения образованного числа в функции $\operatorname{Pr}=3(\mathrm{Y}+0,3333 \ldots)$, Где Y четные числа из ряда ожидаемых простых чисел (Pr), нужно получить корень квадратный из всех образованных чисел и исключить те числа, значение корня квадратного есть целое (при небольших значениях простое число) без остатка......эти числа сложные, как квадраты (вторая степень), простые в большей степени...можно это утверждение отобразить так:

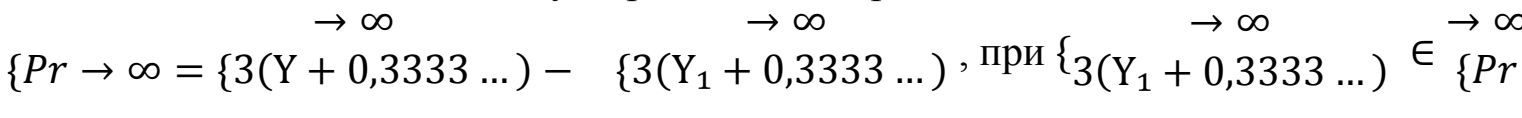

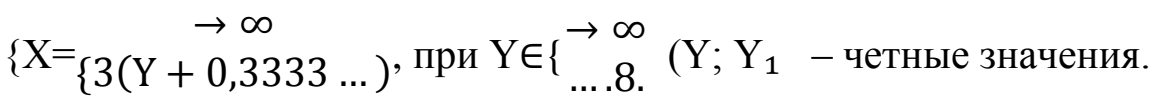

Значения «Ү», оканчивающиеся на «8», образуют сложные «А» группы, поэтому не используются для получения простых чисел. Обозначено как множество $\left\{\begin{array}{l}\rightarrow \infty \\ . . .8 .\end{array}\right.$ Как сложные, не квадратичные числа (квадраты и большие степени простых), образованы следующие: 91; 133; 187; 217; 247; 259; 301; 367; 403; 409; 427; 451; 469; $481 ; 493 ; 511 ; 517, \ldots$.

Рассмотрим образование чисел.

$$
\begin{aligned}
& 3(2+0,3333 \ldots)=7 ; 3(4+0,3333 \ldots)=13 ; 3(6+0,3333 \ldots)=19 ; 3(8+0,3333 \ldots)=25 \in A_{i} \\
& 3((10+0,3333 \ldots)=31 ; 3(12=0,3333 \ldots)=37 ; 3(14+0,3333 \ldots)=53 ; 3(16+0,3333 \ldots)=49 \text {; } \\
& 3(20+0,3333 \ldots)=61 ; 3(22+0,3333 \ldots)=67 ; 3(24+0,3333 \ldots)=73 ; 3(26+0,3333 \ldots)=79 \text {; } \\
& \underline{3(30+0,3333 \ldots)=81} ; 3(32+0,3333 \ldots)=103 ; 3(36+0,3333 \ldots)=109 ; \underline{3(40+0,3333 \ldots)=121} \text {; }
\end{aligned}
$$

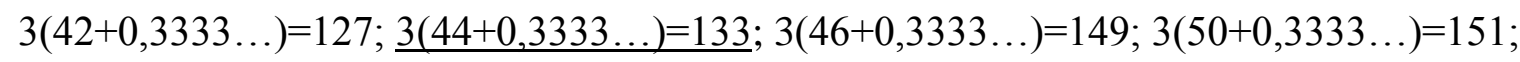

$$
\begin{aligned}
& 3(52+0,3333 \ldots)=157 ; 3(54+0,3333 \ldots)=163 ; 3(56+0,3333 \ldots)=169 ; 3(60+0,3333 \ldots) 181 \text {; } \\
& 3(62+0,3333 \ldots)=187 ; 3(64+0,3333 \ldots)=193 ; 3(66+0,3333 \ldots)=199 ; 3(70+0,3333 \ldots)=211 \text {; } \\
& \underline{3(72+0,3333 \ldots)=217} ; 3(74+0,3333 \ldots)=223 ; 3(76+0,3333 \ldots)=229 ; 3(80+0,3333 \ldots)=241 \text {; } \\
& \underline{3(82+0.3333 \ldots)=247} ; 3(84+0,3333 \ldots)=253 ; \underline{3(86+0,3333 \ldots)=259} ; 3(90+0,3333 \ldots)=271 \text {; } \\
& 3(92+0,3333 \ldots)=277 ; 3(94+0,3333 \ldots)=283 ; 3(96+0,3333 \ldots)=289 ; 3(100+0,3333 \ldots)=301 \text {; } \\
& 3(102+0,3333 \ldots)=307 ; 3(104+0,3333 \ldots)=313 ; 3(106+0,3333 \ldots)=319 \ldots \\
& 3(112+0,3333 \ldots)=337 ; 3(114+0,3333 \ldots)=343 ; 3(116+0,3333 \ldots)=349 \text {; }
\end{aligned}
$$

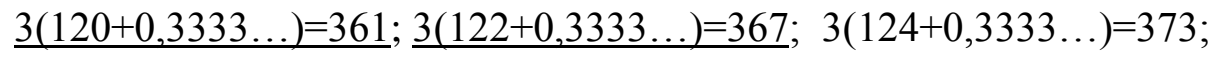

$$
\begin{aligned}
& 3(126+0,3333 \ldots)=379 ; 3(130+0,3333 \ldots)=391 ; 3(132+0,3333 \ldots)=397 \text {; } \\
& \underline{3(134+0,3333 \ldots)=403} ; \underline{3(136+0,3333 \ldots)=409} ; 3(140+0,3333 \ldots)=421 \text {; }
\end{aligned}
$$


$\underline{3(142+0,3333 \ldots)=427} ; 3(144+0,3333 \ldots)=433 ; 3(146+0,3333 \ldots)=439 ;$

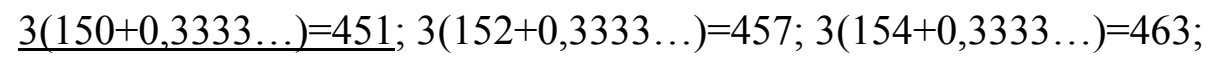

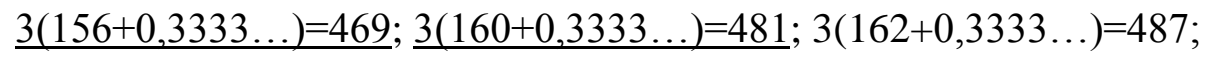

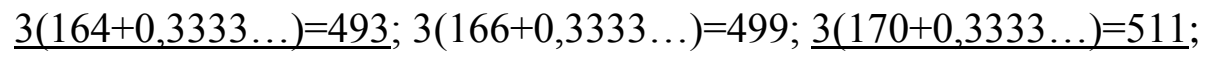

$\underline{3(172+0,3333 \ldots)=517}$

Проверим некоторые значения на деление, принадлежность к сложным...511:7=73;

517:11=47 (образованы произведением простых).

$3(174+0,3333 \ldots)=523 ; \underline{3(176+0.3333 \ldots)=529 ;} \sqrt{529}=23 ; 3(180+0,3333 \ldots)=541$;

$3(182+0,3333 \ldots)=547$.

Как сложные, не квадратичные числа, образованы следующие: 91; 133; 187; 217; 247; $259 ; 301 ; 367 ; 403 ; 409 ; 427 ; 451 ; 469 ; 481 ; 493 ; 511 ; 517 \ldots$ Видна закономерность: в функции $\operatorname{Pr}=3(Y+0,3333 \ldots)$, при значениях «У», оканчивающихся на «0», образуются числа, оканчивающиеся на «1». Среди них могут быть и квадраты простых чисел.

При значениях «Ү», оканчивающихся на «6», образуются числа, оканчивающиеся на «9». Среди них также могут быть квадраты простых чисел $\left(\mathbf{P r}^{2}\right)$.

Простые числа может образовывать также функция $\mathrm{Pr}=5(\mathrm{Y}+0,6666 \ldots)$ при $\mathrm{Y}-$ четных. В этой функции «образующим» остатком $\mathrm{I} \overrightarrow{\mathrm{a}} \mathrm{I}$ есть $0,6666 \ldots$ К примеру: $5(2+0,6666 \ldots) \approx 13 ; 5(4+0,6666 \ldots) \approx 23 ; 5(6+0,6666 \ldots) \approx 33$ (сложное); $5(8+0,6666 \ldots)$ $\approx 43 ; 5(10+0,6666 \ldots) \approx 53 ; 5(12+0,6666 \ldots) \approx 63 ;$ (сложное, условно « $\mathbf{S} ; ; 5(14+0,6666 \ldots)$ $\approx 73 ; 5(16+0,6666 \ldots) \approx 83 ; 5(18+0.6666 \ldots) \approx 93(\in » \mathrm{~S} \gg$ - принадлежит к сложным). $5(20+0,6666 \ldots) \approx 103 ; 5(22+0,6666 \ldots) \approx 133 ; 5(24+0,6666 \ldots) \approx 123(\mathrm{~S}) ; 5(26+0,6666 \ldots$ )$\approx 133 ;(\mathrm{S}) ; 5(28+0,6666 \ldots) \approx 143 ;(\mathrm{S}) .5(30+0,6666 \ldots) \approx 153 ;(\mathrm{S}) .5(32+0,6666 \ldots)=163$; $5(34+0,6666 \ldots) \approx 173 ; 5(36+0,6666 \ldots) \approx 183 ;(S) .5(38+0,6666 \ldots) \approx 193$; $5(40+0,6666 \ldots) \approx 203 ;(\mathrm{S}) .5(42+0,6666 \ldots) \approx 213$; (S). $5(44+0,6666 \ldots) \approx 223$; $5(46+0,6666 \ldots) \approx 233 ; 5(48+0,6666 \ldots) \approx 243 ;(\mathrm{S}) .5(50+0,6666 \ldots) \approx 253$; $5(52+0.6666 \ldots) \approx 263 ; 5(54+0,6666 \ldots) \approx 273 ;(S) .5(56+0,6666 \ldots) \approx 283 ; 5(58+$ $0,6666 \ldots) \approx 293 ; 5(60+0,6666 \ldots) \approx 303 ;(\mathrm{S}) .5(62+0,6666 \ldots) \approx 313 ; 5(64+0,6666 \ldots) \approx$ $323 ; 5(66+0,6666 \ldots) \approx 333 ;(\mathrm{S}), 5(68+0,6666 \ldots) \approx 343 ; 5(70+0,6666 \ldots) \approx 353$; $5(72+0,6666 \ldots) \approx 363 ; 5(74+0,6666 \ldots) \approx 373 ; 5(76+0,6666 \ldots) \approx 383 ; 5(78+0,6666 \ldots) \approx$

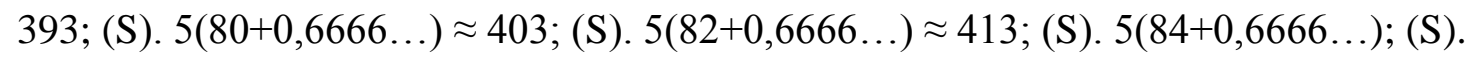
$5(86+0,6666 \ldots) \approx 433 ; 5(88+0,6666 \ldots) \approx 443 ; 5(90+0,6666 \ldots) \approx 453 ;(\mathrm{S})$. $5(92+0,6666 \ldots) \approx 463 ; 5(94+0,6666 \ldots) \approx 473 ;(\mathrm{S}) .5(96+0,6666 \ldots) \approx 483 ;(\mathrm{S})$.

В функции $\operatorname{Pr}=5(\mathrm{Y}+0,6666 \ldots)$ при $\mathrm{Y}=6 ; 12 ; 18 ; 24 ; 30 ; 36 ; 42 ; 48 ; 54 ; 60 ; 66 ; 72 ; 78$; 84; 90; 96 (и других значениях «Ү»), - вместо ожидаемых простых чисел образуются сложные, то есть это смешанная функция числообразования... сложные нечетные числа, в зависимости от числового корня, их образующего, условно можно разделить 
на много групп (и подгрупп). Основные группы с минимальными корнями это: S»3» (корнем является «3», умноженное на нечетное определенное число. Включает подгруппу $\mathrm{S»33»} \mathrm{(образование} \mathrm{сложных} \mathrm{чисел} \mathrm{рассматривается} \mathrm{от} \mathrm{«33»...} \mathrm{далее}$ следуют группы $\mathrm{S} » s »($ или группа $\mathrm{A}$ - группа); $\mathrm{S} » 7 » ; \mathrm{S} » 11 » ; \mathrm{S}$ »13» и другие, о которых будет указано.

При указанных значениях «Ү» от 6 до 96 образуются сложные числа подгруппы $\mathrm{S} » 33 »=33+30 \mathrm{xn}$ (входит в группу $\mathrm{S} » 3 »)$. Все эти числа делятся на «3». 33:3=11 $\in \mathrm{Pr}$; 63:3=21(S); 93:3=31 $\in \operatorname{Pr} ; 123: 3=41 \in \operatorname{Pr} ; 153: 3=51 \in \mathrm{S} » 3 » ; 183: 3=61 \in \operatorname{Pr} ; 213: 3=71 \in \operatorname{Pr}$; 243:3=81(S); 273:3=91(S); 303:3=101 $\in \operatorname{Pr} ; 333: 3=111(\mathrm{~S}) ; 363: 3=121(\mathrm{~S}) ; 393: 3=131(\mathrm{Pr})$; 423:3=141(S); 453:3=151(Pr).

Деление на «3» чисел $\mathrm{S}$ «33» образует простые и сложные числа: квадраты простых чисел $\left(121=11^{2}\right)$; сложные $S \ll 7 »-$ группы: $S \ll 7 »=7+14 x n:(91=7+14 x 6)$; $231=7+14 x 16 \ldots$ Числа при $\mathrm{Y}=6 \mathrm{xn}(\mathrm{n} \rightarrow \infty)$, цельноделимые на «3», в функции $\operatorname{Pr}=5(\mathrm{Y}+0,6666 \ldots)$ для нахождения простых чисел исключаются, как и другие сложные...

Рассмотрим далее способы идентификации и исключения сложных чисел для определения простых чисел... Одним из способов определения простых чисел есть идентификация через группу сложных $\mathrm{S} \ll 7 »=7+14 \mathrm{xn} ; \mathrm{n}=0 \rightarrow 7 \ldots$ В группе образуются числа: $21(\mathrm{n}=1) ; 35(\mathrm{n}=2) ; 49\left(\mathrm{n}=3 ; 49=7^{2}\right) .63 ; 77 ; 91 ; 105 ; 119 ; 133 ; 147 ; 161 ; 175 ; 189$; $203 ; 217 ; 231 ; 245 ; 259 ; 273 ; 287 ; 301 ; 315 ; 329 \ldots$

Разделим значения образуемых чисел в группе на «7», получим: $1 ; 3 ; 5 ; 7 ; 9 ; 11 ; 13$; $\underline{15} ; 17 ; 19 ; \underline{21} ; 23 ; \underline{25} ; 27 ; 29 ; 31 ; \underline{33} ; \underline{35} ; 37 ; 39 ; \ldots$ то есть ряд всех нечетных чисел функции $\mathrm{Y}=3+2 \mathrm{xn}(\mathrm{n}=0 \rightarrow 3 ; \mathrm{n}=1 \rightarrow 5)$; «1» образуется при $\mathrm{n}=-1$, поэтому не учитывается... Сложные подчеркнуты.

Таким образом, в функции $S$ «3»=9+6xn, - образуются только сложные нечетные числа...

Если значение функции (S «7»=7+14xn) - разделить на «7» и исключить из множества образованных чисел значения чисел функции $S \ll 3 »=9+6 \mathrm{xn}$, получим огромное множество простых чисел до бесконечности. При этом надо исключить и все сложные функции $S \ll 7 »=7+14 \times n$ (n> 0).

Вывод: $\left\{\operatorname{Pr} \rightarrow \infty=\left\{\rightarrow \infty \frac{7+14 x n}{7}-\{\rightarrow \infty 7+14 \times n,(n>0)-\{\rightarrow 9+6 n\right.\right.$;

Или коротко: $:\left\{\boldsymbol{P r} \rightarrow \infty=\left\{\rightarrow \infty \frac{S \text { "7" }}{7}-\{\rightarrow \infty \boldsymbol{S}\right.\right.$ "7" - $\{\rightarrow \infty \boldsymbol{S}$ "3". Это первый вариант определения простых. 
Символ $\{\rightarrow \infty \mathrm{X}$ - означает , что множество «Х» стремится к бесконечности...

Рассмотрим деление и образование результата для значения функции $\mathrm{S} \ll 3 » .9: 3=3$; $15: 3=5 ; 21: 3=7 ; \ldots .1 ; 3 ; 5 ; 7 \in \operatorname{Pr}$ (простые числа). 27:3=9; 33:3=11; 39:3=13; 45:3=15; $51: 3=17 ; 57: 3=19 ; 63: 3=21 ; 69: 3=23 ; 75: 3=25 ; 81: 3=27 ; 87: 3=29 ; 93: 3=31 ; 99: 3=33$; $105: 3=35 ; 111: 3=37 ; 117: 3=39 ; .11 ; 13 ; 17 ; 19 ; 23 ; 29 ; 31 ; 37$, - простые числа; $15 ; 45$; 25; 75; 105, - сложные одновременно двух групп - S «3» и $\mathrm{S} « 5 »$ (А - группа.) 33; 99 $\in \mathrm{S}$ «3» и $\mathrm{Z}$ - гомогенной группе (такие числа во всех значениях одинаковы. $K$ примеру: 1111; 333; 5555; 7777). Всегда сложные, без остатка, делятся на «11».

Числа, образуемые одновременно в двух группах, называются объединяющими или


Учитывая, что при делении на «3» чисел $\mathrm{S} « 3 »-$ группы образуется множество простых чисел, их можно определять так:

$\left\{\rightarrow \infty \operatorname{Pr}\right.$ или $\left\{\operatorname{Pr} \rightarrow \infty=\left\{\frac{S " 3 "}{3} \rightarrow \infty-\{\mathrm{S} \ll 3 » \rightarrow \infty-\mathrm{A}-\mathrm{Z}\right.\right.$ (определение А и Z групп не представляет проблемы).

Это второй способ определения простых чисел до бесконечности. Рассмотрим деление сложных $\mathrm{S}$ «3» - группы по возрастающей...

$123: 3=41 ; 129: 3=43 ; 135: 3=45 ; 141: 3=47 ; 147: 3=49 ; 153: 3=51 ; 159: 3=53 ; 165: 3=55$; $171: 3=57.41 ; 43 ; 53 \in \operatorname{Pr} ; 49=7^{2} \in S \ll 7 » ; 45 ; 51 ; 57 \in \mathrm{S} \ll 3 »$. Видно, что в значении (S «3»:3) кроме простых чисел и сложных $S \ll 3 »$ образовалось и сложное $S \ll 7 », 7^{2}=49$. Квадраты простых чисел образуются и в больших значениях (S «3»:3) до

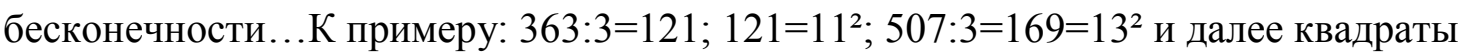
всех простых чисел, до бесконечности. В значениях ( $\mathrm{S} « 3 »: 3)$ образуются сложные нечетные числа больших порядков, по возрастающей. К примеру: 663:3=221; $221=13 \times 7 ; \in \mathrm{S} « 13 » \ldots$ От числа $187=11 \times 17$ (сложное $\mathrm{S} » 11 ») ; 561: 3=187,-$ можно составить закономерность: $\quad \mathrm{Y}=\mathrm{X} \pm 22 \mathrm{xn} \rightarrow \mathrm{S} \ll 11 »$ (образуются сложные, делимые на «11»). При уменьшении на «22» образуются числа: 187-22=165; далее образуются $143 ; 121\left(11^{2}\right) ; 99 ; 77 ; 55 ; 33 ; 11$. При увеличении на «22» от «187» образуются числа: $187+22=209 ; 231 ; 253 \in \operatorname{Pr} ; 275 ; 297 ; 319 \in \operatorname{Pr} ; 341 ; 363 ; 385 ; 407 \in \operatorname{Pr}$; $413 ; 435 ; 457 \in \operatorname{Pr} .$. В группе $\mathrm{S} « 11 »$ образуются объединяющие числа: $\mathrm{A} \leftarrow 55 \rightarrow S$ «11»; А $\leftarrow 165 \rightarrow S « 11 » ;$ образуются и простые числа. Значит, функция $\mathbf{Y = 1 1 + 2 2 x n}$ есть функцией смешанных чисел... Tаким образом, в функции $\mathrm{S}$ « $3 / 3 »=(9+6 \mathrm{xn}): 3=3+2 \mathrm{n}(\mathrm{n} \rightarrow \infty)$ образуются простые числа методом исключения из образованных чисел значений функции $\mathrm{Y}=9+6 \mathrm{xn}$ (сложные), $\mathrm{A}-$ группы ( $\mathrm{S}$ «»)), Z группы (гомогенные числа), а также новые значения, не образованные в функции $\mathrm{Y}=7+14 \mathrm{xn}$ и $\mathrm{Y}=9+6 \mathrm{xn}$.

Не определяются таким способом такие простые, как 253; 319; 407; 457 и другие. Но он есть более полный, чем второй, учитывая исключение D - чисел (числа, не 
образованные как сложные в группах $\mathrm{S}$ «3» и $\mathrm{S}$ «7»; A и Z, образовавшиеся в $\mathrm{Y}=3+2 \mathrm{xn})$.

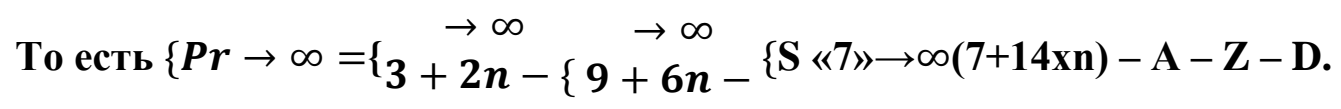

Ряд всех нечетных чисел, начиная с «1» обозначим $\mathbf{Q}_{\mathbf{1}}$; от значения первого сложного числа «9»- $\mathbf{Q}_{2}$. В ряду $\mathrm{Q}_{1}$ значения всех чисел вычисляются по формуле $\mathrm{X}=2 \mathrm{n}_{1}-1$; в ряду $\mathrm{Q}_{2}$ по формуле $\mathrm{X}=7+2 \mathrm{xn}_{2} ;\left(\mathrm{n}_{2}=1 \rightarrow 9 ; \mathrm{n}_{2}=2 \rightarrow 11 ; \mathrm{n}_{2}=3 \rightarrow 13\right.$; $\mathrm{n}_{2}=4 \rightarrow 15 ; \mathrm{n}_{2}=5 \rightarrow 17 ; \mathrm{n}_{2}=6 \rightarrow 19 ; \mathrm{n}_{2}=7 \rightarrow 21 ; \mathrm{n}_{2}=8 \rightarrow 23$ и далее до $\left.\infty\right)$.

Значения «X» на «+8» больше в функции $\mathrm{X}=7+2 \mathrm{xn}_{2}$, чем в функции $\mathrm{X}=2 \mathrm{n}_{1}-1$, при $\mathrm{n}_{2}=\mathrm{n}_{1}$. В функции $\mathrm{Q}_{2}$, начиная с «11», образуется множество простых чисел с промежуточными сложными , увеличиваясь от «11» на «+2xn» значений...

Рассмотрим образование квадратов нечетных чисел в функции $\mathrm{X}=7+2 \mathrm{xn}_{2}, \mathrm{c}$ «11»... $\mathrm{X}=7+2 \mathrm{xn}_{2}=(11+2 \mathrm{xn})^{2} ; 7+2 \mathrm{xn}_{2}=121+44 \mathrm{xn}+4 \mathrm{xn}^{2} ; 2 \mathrm{n}_{2}=121+44 \mathrm{xn}+4 \mathrm{n}^{2}-7$; $\mathrm{n}_{2}=57+22 \mathrm{xn}+2 \mathrm{xn}^{2}$; заменим $\mathrm{n} 2$ на «Y».. Y=57+22xn+2xn ${ }^{2}$. При $\mathrm{n}=1 \rightarrow 57+22+2=57+24=81.81=9^{2}=3^{4} \ldots 3 \in \operatorname{Pr} \ldots \ll 57 »-$ му порядку в ряду $\mathrm{Q}_{2}$ соответствует $121=11^{2} ; 11 \in \operatorname{Pr} . .24$-му порядку в $\mathrm{Q}_{2}$ соответствует сложное А-группы «55», - (всем n порядкам в функции квадратов нечетных, с окончанием на ....4, отвечают сложные А-группы. 81-му порядку отвечает $169=13^{2} ; 13 \in \operatorname{Pr}(81 \rightarrow 169)$. Значения $\mathrm{Y}=57+22 \mathrm{xn}+2 \mathrm{n}^{2}$ и $\mathrm{Y}_{1}=22 \mathrm{n}+2 \mathrm{n}^{2}$, - определяют $\mathrm{n}-$ порядки сложных чисел в функции $\mathrm{Q}_{2}=7+2 \mathrm{xn}_{2} \ldots \mathrm{n}-$ порядки в функции квадратов также с окончанием на «...9 9 », как и «....4», - образуют в $\mathrm{Q}_{2}$ числа $\mathrm{A}$ - группы. При $\mathrm{n}=2 \rightarrow 57+44+8=57+52=109 ; \mathrm{n}_{2}=52 \rightarrow 111 \in S$ "3"; $109 \rightarrow 325 \in \mathrm{A} ; 109 \in \operatorname{Pr} ;$ при $\mathrm{n}=3 \rightarrow 57+66+18=57+84=141 ; \mathrm{n}_{2}=84 \rightarrow 7+2 \times 84=175 \in \mathrm{A} ; \mathrm{n}_{2}=175 \rightarrow 357 \in \in \mathrm{S} \ll 3 » ; \in \mathrm{S} \ll 7 »$ (совместное число). $\mathbf{S} \ll \mathbf{3} » \leftarrow \mathbf{3 5 7} \rightarrow \mathbf{S} « 7 » . . . \mathrm{n}_{2}=141 \rightarrow 289=17^{2}(17 \in \mathrm{Pr})$

$\mathrm{n}_{2}=289 \rightarrow 7+2 \times 289=7+578=585 \in \mathrm{A}$; $\mathrm{n}=4 \rightarrow 57+22 \times 4+2 \times 16=57+120=177 \ldots \mathrm{n}_{2}=120 \rightarrow 7+240=247(247: 13=19)$; $\mathrm{n}_{2}=177 \rightarrow 361=19^{2} ; \mathrm{n}_{2}=361 \rightarrow 729=27^{2}$.

$\mathrm{n}_{2}=729 \rightarrow 1465 \in \mathrm{A} ; \mathrm{n}_{2}=1465 \rightarrow 2937 \in \mathrm{S} \ll 3 »(2937: 3=979) ; \mathrm{n}=5$ $\rightarrow 57+110+50=57+160=217 ; \mathrm{n}_{2}=160 \rightarrow 7+2 \times 160=327 \in \mathrm{S} \mathrm{S} \ll 3 » . \mathrm{n}_{2}=217 \rightarrow 441=21^{2}$; $\mathrm{n}=6 \rightarrow 57+132+72=57+204=261 ; \mathrm{n}_{2}=204 \rightarrow 415 \in \mathrm{A} ; \mathrm{n}_{2}=261 \rightarrow 529=23^{2} ; 23 \in$ $\operatorname{Pr} ; \mathrm{n}=7 \rightarrow 57+154+98=57+252=309 ; \mathrm{n}_{2}=252 \rightarrow 511 \in S \ll 7 \gg(511: 7=73) ; \mathrm{n}_{2}=309 \rightarrow 625=25^{2} \in$ А... Функция порядков сложных значений $\mathrm{Y}=57+22 \mathrm{xn}+2 \mathrm{xn}^{2}$, - позволяет определить $\mathrm{n}$ - порядки сложных чисел в функции $Q_{2}=7+2 \mathrm{xn}_{2} \ldots$

Существует закономерность: половина значения квадратов нечетных чисел с окончанием «....1,» округленных до целых значений, чаще есть простые числа... 
$81: 2=40,5 \approx 41 \in \operatorname{Pr} ; 121: 2=60,5 \approx 61 \in \operatorname{Pr} ; 441: 2=220,5 \approx 221=11^{2}$ ( или квадраты, четные степени простых чисел, $\operatorname{Pr}^{\mathrm{n}},{ }^{\mathrm{n}}-$ четное число).

Учитывая функцию образования порядков сложных чисел, можно расширить

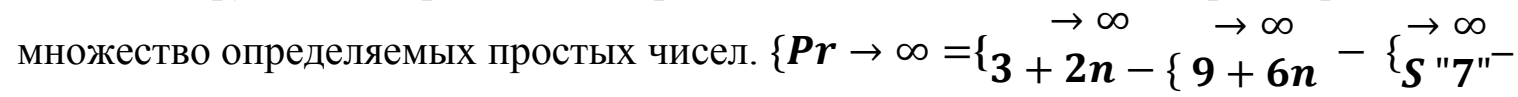
$A-Z-D-\left(\right.$ сложные в $Q_{2}=7+2 x_{2}$, где $\left.n_{2}=57+22 x n+2 x^{2} ; n_{2}=22 x n+2 n^{2}\right) \ldots$ Частично простые числа определяются делением, половиной значения квадратов нечетных.

Образование простых чисел в группе простых

$\operatorname{Pr} \ll 13 »=13+10 \times n$ $(\mathbf{n}=\mathbf{0} \rightarrow \mathbf{1 3} ; \mathbf{n} \rightarrow \infty)$. Интервал между образуемыми ближайшими числами есть «10»: 13, $23 ; \underline{33} ; 43 ; 53 ; 63 ; 73 ; 83 ; \underline{93} ; 103 ; 113 ; \underline{123} ; 133 ; \underline{143} ;(143: 11=13) ; 153 ; 163$;

$173 \ldots$ (сложные подчеркнуты)... В данной группе образуются и сложные подгруппы $\mathrm{S} « 33 »=33+30 \mathrm{xn}, \in S$ "3"(входит в группу сложных $S$ "3)...

В функции $\operatorname{Pr} « 13 »$ образуются такие же числа, как и в функции $\operatorname{Pr}=\mathbf{5}(\mathbf{Y}+\mathbf{0 , 6 6 6 6 . . . )})$ Значит, $5(\mathrm{Y}+0,6666 \ldots)=13+10 \mathrm{xn}$ (но в функции $5(\mathrm{Y}+0,6666 \ldots)$ простых других значений образуется больше. Это числа: $5 ; 11 ; 17 ; 23 ; 29 ; \underline{35} ; 41 ; 47 ; 53 ; 59 ; \underline{65} ; 71 ; \underline{77}$; $83 ; 89 ; \underline{95} ; 101 ; 107 ; 113 ; \underline{119} ; \underline{125 ; 131, \ldots+6 x n . . . ~ П о д ч е р к н у т ы ~ с л о ж н ы е ~ А ~-~ г р у п п ы, ~}$ $\mathrm{Z}$ - группы (77); S «7» (119)... Далее идут: 137; 143(сложное, 143:11=13) 149; 155; 161; $\in$ S "7"; 167 ; 173; 179; 185; 191; 197; 203; $209 \in S$ "11"; 215; 221; (112; $221=13 x 17 ;$ сложное); 227; 233,... Аналогично можно определить простые числа от «7» в функции $\mathbf{P r}=\mathbf{7 + 6 x n}$, где «7» соответствует n= 0. Это числа: 7; 13; 19; 25; 31; 37; $43 ; \underline{49} ; \underline{55} ; 61 ; 67 ; 73 ; 79 ; \underline{85} ; \underline{91} ; 97 ; 103 ; 109 ; \underline{115} ; \underline{121} ; 127 ; \underline{133} ; 139 ; \underline{145} ; 151 ; 157$;

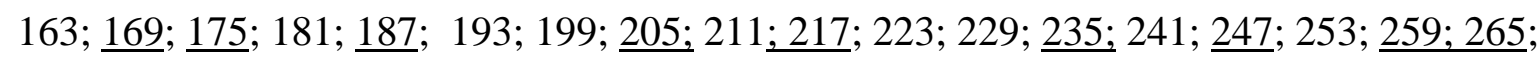
$271 ; 277 ; 283 ; \underline{289}, \ldots$ (сложные подчеркнуты)...

В функции $\operatorname{Pr}$ «7» образуются и сложные числа, в частности, квадраты простых чисел: $121=11^{2} ; 169=13^{2} ; 289=17^{2} \ldots$ Рассмотрим, квадраты каких чисел образуются в функции $\operatorname{Pr} \ll 7 »=7+6 x n$ n $\rightarrow \infty$.

Применим простые и сложные нечетные числа для получения их квадратов...

Квадраты простых чисел: $23^{2}=529 ; 31^{2}=961 ; 29^{2}=841 ; 37^{2}=1369 ; 41^{2}=1681 ; 43^{2}=1849$; $47^{2}=2209 ; 53^{2}=2809 ; 59^{2}=3841 ; 61^{2}=3721 ; 67^{2}=4489 \ldots$

Рассмотрим их образование в функции $\operatorname{Pr} « 7 »=7+6 x n . . .7+6 \mathrm{n}=529 ; \mathrm{n}=87 ; \operatorname{Pr} « 7 »=961$; $\mathrm{n}=159 ; \quad \operatorname{Pr} \ll 7 »=841 ; \mathrm{n}=139 ; \operatorname{Pr} \ll 7 »=1369 ; \mathrm{n}=227 ; \operatorname{Pr} \ll 7 »=1681 ; \mathrm{n}=279 ; \operatorname{Pr}$ $\langle 7 »=1849 \rightarrow \mathrm{n}=307 ; \operatorname{Pr}\langle 7 »=2209 \rightarrow \mathrm{n}=367 ; \operatorname{Pr}\langle 4 »=2809 \rightarrow \mathrm{n}=467 ; \operatorname{Pr}\langle 7 »=3481 \rightarrow \mathrm{n} 579 ; \operatorname{Pr}$ $« 7 »=3721 \rightarrow \mathrm{n}=619 ; \operatorname{Pr} \ll 7 »=4489 \rightarrow \mathrm{n}=747 \ldots$

Вывод: образуются целые n - значения (порядки), значит, в функции Pr «7»= 7+6xn n $\rightarrow \infty$, квадраты простых чисел образуются... Применим сложные нечетные 
числа для образования квадратов...21 $21^{2}=441 ; 27^{2}=729 ; 25^{2}=625 ; 33^{2}=1089 ; 35^{2}=1225$; $39^{2}=1521 ; 45^{2}=2025 ; 49^{2}=2401 ; 51^{2}=2601 ; 55^{2}=3025 ; 57^{2}=3249 ; 63^{2}=3969 ; 65^{2}=4225 \ldots$

Определим их $\mathrm{n}$ - значения в функции $\operatorname{Pr} « 7 » . \operatorname{Pr} « 7 »=7+6 \mathrm{xn}=21^{2}=441 ; \mathrm{n}=72,3333 \ldots$ Pr $\langle 7 »=625 \rightarrow \mathrm{n}=103 ; \operatorname{Pr}\langle 7 »=729 \rightarrow \mathrm{n}=120,3333 \ldots ; \operatorname{Pr}\langle 7 »=1089 \rightarrow \mathrm{n}=180,3333 \ldots \operatorname{Pr} \ll 7 »=$ $1225 \rightarrow \mathrm{n}=203 ; \quad \operatorname{Pr} \ll 7 »=1521 \rightarrow \mathrm{n}=252,3333 \ldots ; \operatorname{Pr} \ll 7 »=2401 \rightarrow \mathrm{n}=339 ;\left(2401=49^{2}=7^{4}\right)$; $\operatorname{Pr} \ll 7 »=2601 \rightarrow \mathrm{n}=432,3333 \ldots ; \quad \operatorname{Pr} \ll 7 »=3025 \rightarrow \mathrm{n}=503 ; \operatorname{Pr}$ $\ll 7 »=3249 \rightarrow \mathrm{n}=540,3333 \ldots ; \operatorname{Pr} \ll 7 »=3969 \rightarrow \mathrm{n}=660,3333 \ldots ; \operatorname{Pr} \ll 7 »=4225 \rightarrow \mathrm{n}=703 \ldots$

Видно, что все $\mathrm{n}$ - порядки с окончанием «...3», - в функции $\operatorname{Pr} « 7 »$ образуют сложные A - группы. На примере $\operatorname{Pr} « 7 »=2401 \rightarrow \mathrm{n}=339 ;\left(2401=49^{2}=7^{4}\right)$ видно, что целые n - порядки квадратов сложных чисел в функции Pr «7»=7+6xn (смешанной) образуются в том случае, если само сложное число образовано минимальным значением, как квадратом простого $\left(49=7^{2}\right)$; то есть целый n порядок возможен при значении $\operatorname{Pr}$ «7», как четной степени простого числа. $\left(2401=49^{2}=7^{4}\right) \ldots$

Первым этапом определения простых чисел является исключение сложных А -

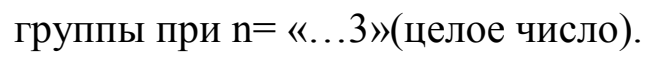

Вторым этапом есть извлечение корней квадратных из образуемых в функции $\operatorname{Pr}$ “7” чисел, от меньших значений к большим...целые числа получатся при извлечении корней квадратных.

Корней квадратных из чисел, образованных возведением простых в степени: 2; 4; 6; $8 ; 10 ; 12 ; 14 ; 16 \ldots$ - в четную $2 \mathrm{xn}-$ степень. Значит, $\operatorname{Pr}=\sqrt[2 n]{\mathrm{Y}}=\mathbf{X}$ - целое число... Числа, образовавшиеся с остатком при получении $\sqrt[2 n]{S}$, - говорят о том, что в значении $\mathbf{S}$ находится нечетная степень простого числа... При небольших относительно Y - значениях определяются корни, простые числа, образующие сложные возведением простых в степени $2 \mathrm{xn} . .$.

Правило проверки числа, полученного как корень квадратный из числа «Ү», - к принадлежности к простым числам. Число «Х», как предполагаемое Рт (простое), следует проверить, как «Ү» в функции $\mathrm{Y}=7+6 \mathrm{xn} . .$. Если образуется целый порядок «n», тогда число или простое в любой степени (сложное), то есть из «Ү» надо также извлечь корень квадратный (для уверенности, что значение есть простым)... Числа «Y», не образованные как степени квадратные, - могут образовываться в «Рr», как нечетные степени простых.

\section{Поэтому простыми числами есть те, из которых в данной функции}

\section{1. Извлекается корень с остатком.}

2. В функции $\mathrm{Y}=7+6 \mathrm{xn}$, - образуется целый «n» порядок. $\oint \operatorname{Pr} \rightarrow \sqrt{\operatorname{Pr}}=\mathrm{X}, \ldots ; \operatorname{Pr}=$ $7+6 \mathrm{xn}, \mathrm{n}-$ целое. Данная закономерность проявляется для чисел, образуемых в 
функции $\operatorname{Pr} « 7 »=7+6 x n$ (n - порядки, равные 7xn, где $\mathrm{n}$ - четное число, образуют сложные числа).

Для определения множества простых чисел из более крупных $\mathrm{Y}=7+6 \mathrm{xn}$, следует извлекать $\sqrt[4]{\mathrm{Y}} ; \sqrt[6]{\mathrm{Y}} ; \sqrt[8]{\mathrm{Y}}$ и далее по возрастающей, (корни четвертой степени от $100,-$ для исключения сложных; параллельно проверять $\mathrm{n}-$ значения в $\operatorname{Pr}$ «7» значений четных корней... Образование значений четных корней с остатком при «n» целых порядках в Y = 7+6xn, - указывает на простое число в значениях $\mathrm{Pr}=\mathrm{X}=$ $\sqrt[a]{7+6 \times n} 4 ; a-$ четная степень; $X=7+6 x n$ (n - целое число). Примеры: $\mathrm{n}=1 \rightarrow 7$ $=6 \mathrm{xn}=13 ; \sqrt{13}=3,60555 \ldots ; 13 \in \operatorname{Pr} ; \mathrm{n}=2 \rightarrow 7+6 \times 2=19 ; \sqrt{19}=4,358898 \ldots ; 19 \in \operatorname{Pr} ; \mathrm{n}=$ $3 \rightarrow 7+6 \times 3=25 ; \quad \sqrt{25}=5 \in \mathrm{A}$; все $\mathrm{n}-$ порядки с окончанием на «...3» образуют сложные числа в $-\mathrm{Y}=7+6 \mathrm{xn} ; \mathrm{n}=23 \rightarrow 7+6 \times 23=145 \ldots$ образуется $\mathrm{A}-$ группа. $\mathrm{n}=27$ $\rightarrow 7+6 \times 27=169 ; \sqrt{169}=13 ; \mathrm{n}=47 \rightarrow 7+6 \times 47=289 ; \sqrt{289}=17$.

Меньшие значения $\sqrt{\mathrm{Y}}=\mathrm{X}, \ldots$ полученные с остатком в фнкции $\mathrm{Y}=7+6 \mathrm{xn},-$ подтверждают, что значение $\mathrm{Y}_{1}=\mathrm{Y}$, образовавшиеся как корни квадратные из больших значений $\mathrm{Y}_{2} ; \mathrm{Y}_{1}=\sqrt{\mathrm{Y}_{2}},-$ есть простые числа, извлеченные из их квадратов, при целых n-порядках значений $\mathrm{Y}_{2}$ в функции $\mathrm{Y}=7+6 \mathrm{xn}$. Если целому числу $\mathrm{Y}_{1}$ соответствует целый $\mathrm{n}_{2}$-порядок в зависимости $\mathbf{Y}_{\mathbf{1}}=\sqrt[a]{\mathrm{Y}_{2}}$, тогда $\mathrm{Y}_{2}$ - значение четной степени $\mathrm{Y}_{1} \ldots$ Простые числа, большинство их, можно определить по формуле:

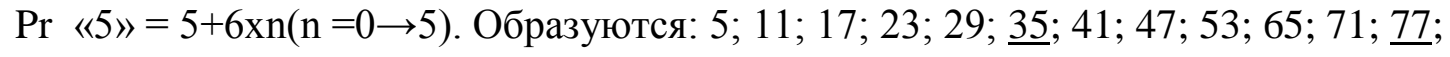
(гомогенное Z число); 83; 89; 95; 101; 107; 113; 119; $\underline{125} ; 131 ; 137 ; \underline{143} ; 149 ; \underline{155} ; 161$;

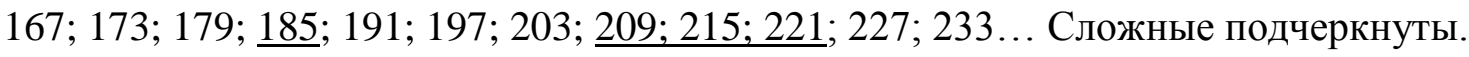

Многие значения не образуемые в $\mathrm{Y}=7+6 \mathrm{xn}$, образуются в функции $\mathrm{Y}=5+6 \mathrm{xn},-17$; $23 ; 41 ; 47$ и другие. Однако в Y = 7+6хn образуются квадраты не образованных простых чисел при целых n-порядках в функции $\operatorname{Pr} « 7 »$. Значит, простые числа из функции $\mathrm{Y}=5+6 \mathrm{xn}$, - подтверждают, что образованные значения $\sqrt{7+6 \mathrm{xn}}=\mathrm{X}-$ целое, $\in \mathrm{Pr}$, если число «Х» входит, образуется функцией $\mathrm{Y}=5+6 \mathrm{xn}$, как $\mathrm{Pr} . . . \mathrm{B}$ функции $Y=7+6 x n$ могут образовываться квадраты сложных трех - и (более) значных чисел, образованные как трех (и более) - значные в функции Y = $5+6 \times n$.

К примеру: $119^{2}=4161 ; \mathrm{Y}=7+6 \mathrm{xn}=14161 ; \mathrm{n}=2359 ; 143^{2}=20449 ; 7+6 \mathrm{n}=20449 ; \mathrm{n}=$ 3407.

Если при целом «n» в функции $\mathrm{Y}=7+6 \mathrm{xn}$, из значения $\mathbf{Y}=\mathbf{X}^{\mathbf{n}}, \mathrm{n}-$ четная степень, не извлекается корень квадратный до получения целого двухзначного $(\sqrt{289}=17$; $\sqrt{361}=19$ ит.д), то квадрат числа, вероятно, образован сложным числом из других функций... К примеру: $\sqrt{143}=11,95826 \ldots ; 143^{2}=20449$, при $n=3407$ в функции $\operatorname{Pr}$ 
«7» или квадрат образован от трехзначного простого числа функции, отличной от Y $=7+6 \times n$.

$137^{2}=18769 ; \mathrm{n}=3127 ; 137 \in \operatorname{Pr} ; \mathrm{n}=21,6666 \ldots 143 \rightarrow \mathrm{n}=22,6666 \ldots \mathbf{n}$ - порядки чисел, образовавших квадраты в значениях функции $Y=7+6 x n$, - как образованных простых и сложных (не квадратичных) чисел в функции $\mathbf{Y}=\mathbf{5 + 6 x n}$. (137ePr; 143 $=11 \times 13)$, в $\mathrm{n}-$ значениях функции $\mathbf{Y}=\mathbf{7 + 6 x n}$, - образуют остаток $0,6666 \ldots$, в отличие от сложных функции $\mathrm{Y}=7+6 \mathrm{xn}$, при которых $\mathrm{n}$ - порядок - целое число... значит, при получении $\mathrm{Y}=\sqrt{7+6 \mathrm{xn}}$, следует проверять $\mathrm{n}-$ порядок образовавшегося числа «Ү», для установления происхождения числа в той или другой функции... $\mathbf{n}-$ порядки квадратов простых чисел в $Y=7+6 \times n$, образующиеся как целые числа, - основа определения Pr...

Рассмотрим образование квадратов нечетных чисел от «9».

$9^{+16} 25^{+24} 49^{+32} 81^{+40} 121^{+48} 169^{+56} 225^{+64} 289^{+72} 361^{+80} \quad 441^{+88} \quad 529 \ldots$.

Видна закономерность, - интервал между квадратами нечетных чисел увеличивается на «+8». Если точкой отсчета квадратов нечетных чисел принять «9» $\left(3^{2}\right)$, то каждое следующее значение квадрата нечетного числа, назовем Q, можно определить по формуле $\mathbf{Q}=\mathbf{9 + 8 x n}(\mathrm{n}=2 \rightarrow 25 ; \mathrm{n}=5 \rightarrow 49 ; \mathrm{n}=9 \rightarrow 81 ; \mathrm{n}=20 \rightarrow 169)$. В Q - значениях наблюдается рост $\mathbf{n}-$ порядков: $2^{+3} 5^{+4} 9^{+5} 14^{+6} 20 \ldots$

Если принять $\mathrm{n}_{1}=2 \rightarrow 25 ; \mathrm{n}_{2}=5 \rightarrow 49$, то $\Delta \mathbf{n}=\mathbf{n}_{\mathbf{2}}-\mathbf{n}_{\mathbf{1}}$ (приращение порядка равно: $5-2$ $=3 ; 9-5=4 ;(4-3=1) ; 20-14=6 ;(6-5=1)$. Приращение $(\Delta \mathrm{n})$ порядка при каждом значении $\mathrm{Q}$ увеличивается на «+1»...

Если $\Delta \mathrm{n}=4$, то $\mathrm{n}_{3}=\mathrm{n}_{2}+\Delta \mathrm{n}=5+4=9 \rightarrow 81$.

$\Delta \mathrm{n}=5 \rightarrow 9+5=14 ; \mathrm{n}_{4}=14 ; \Delta \mathrm{n}=6 \rightarrow 6+14=20 ; \mathrm{n}_{5}=20 ; \Delta \mathrm{n}=7 ; \mathrm{n}_{6}=20+7=27 ;$

$\Delta \mathrm{n}=8 \rightarrow 27+8=35 \ldots$

$\Delta \mathrm{n}=9 \rightarrow 35+9=44 ; \Delta \mathrm{n}=10 \rightarrow 44+10=54 ; \Delta \mathrm{n}=11 \rightarrow 54+11=65 ; \Delta \mathrm{n}=12 \rightarrow 65+12=77 ; \Delta \mathrm{n}$ $=13 \rightarrow 77+13=90 ; \Delta \mathrm{n}=14 \rightarrow 90+14=101 ; \Delta \mathrm{n}=15 \rightarrow 101+15=119$. Значит, порядок квадратов определяется: $\mathrm{n}_{2}=\mathrm{n}_{1}+\Delta \mathrm{n}_{2 \cdot}\left(\Delta \mathbf{n}_{2}=\Delta \mathbf{n}_{1}+1\right.$; приращение каждого $\mathbf{n}-$ значения большего соседнего квадрата нечетного числа увеличивается на «+1».

Можно составить таблицу «n» - порядков квадратов чисел Q, начиная с $9=3^{2} \ldots$ Значения $\mathrm{n}-$ порядков Q: $\mathrm{n}=0 \rightarrow 9 ; \mathrm{n}=2 \rightarrow 25 ; \mathrm{n}=5 \rightarrow 49 ; \mathrm{n}=9 \rightarrow 81 ; 14 \rightarrow 121 ; 20 \rightarrow 169$; $27 \rightarrow 225 ; 35 \rightarrow 289 ; 44 \rightarrow 361 ; 54 \rightarrow 441 ; 65 \rightarrow 529 ; 77 \rightarrow 625 ; 90 \rightarrow 729 ; 104 \rightarrow 841 ;$ $119 \rightarrow 961 ; 135 \rightarrow 1089 ; 152 \rightarrow 1225 ; 179 \rightarrow 1369 ; 189 \rightarrow 1521 ; 209 \rightarrow 1681 ; 230 \rightarrow 1849 ;$ $252 \rightarrow 2025 ; 275 \rightarrow 2209 ; 299 \rightarrow 2401$. 
n=324 $\rightarrow 2601 \ldots$ n - порядки сложных чисел, цельноделимых на «3»,- образуют квадраты сложных нечетных чисел, цельноделимых на «3»... n=90 $\rightarrow 729=$ $27^{2}(90: 3=30 ; 27: 3=9) ; \mathrm{n}=189 \rightarrow 1521=39^{2}(189: 3=39 ; 39: 3=13)$ $\mathrm{n}=324 \rightarrow 2601=51^{2}(324: 3=108 ; 51: 3=17)$.

Еще раз рассмотрим образование квадратов нечетных чисел от «9»...

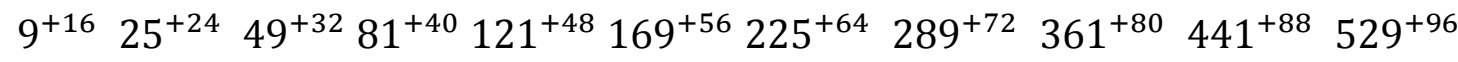
$625^{+104} 729^{\rightarrow \infty}$

$\Delta \mathrm{Q}=104 \rightarrow 729 ; \sqrt{729}=27($ сложное $\mathrm{S} \ll 3 ») ; \Delta \mathrm{Q}=128 \rightarrow 1089 ; \sqrt{1089}=33 \in \mathrm{S} \ll 3 »$.

Увеличение приращения квадрата ближайшего сложного «33» (к 27²) составляет «+24»: $128-104=24$.

От 729 ряд Q следует так: $729^{+112} 841^{+120} 961^{+128} 1089^{+136} 1225 \ldots$

Проверим $\Delta \mathrm{Q}=152 \rightarrow 1521 ; \sqrt{1521}=39 \in \mathrm{S} \ll 3 » ; \Delta \mathrm{Q}=144 \rightarrow 1369 ; \sqrt{1369}=37 ; 1521^{+160}$ $1681(\sqrt{1681}=41)$ От 1681 следуют: $1681^{+168} 1849(\sqrt{1849}=43)^{+176}$

$2025(\sqrt{2025}=45 \in \mathrm{A} \in \mathrm{S} \ll 3 » ; \mathrm{A} \leftarrow 45 \rightarrow \mathrm{S} \ll 3 »$. (число, объединяющее две группы сложных). 176-152=24; 152 $\rightarrow 1521=392 ; 39 \in \mathrm{S} \ll 3 » ;$ от 2025 следуют: $\quad 2025^{+184} 2209$ $(\sqrt{2209}=47)^{+192}-2401\left(\sqrt{2401}=49 ; 49=7^{2}-\text { квадрат простого числа }\right)^{+200} 2601(\sqrt{2601}$ $=51 \in \mathrm{A} \in \mathrm{S} \ll 3 ») \ldots{ }^{+208} 2809\left(\sqrt{2809}=53^{+216} 3025\left((\sqrt{3025}=55 \in \mathrm{A}){ }^{+224}\right.\right.$ $3249(\sqrt{3249}=57 \in S \ll 3 » ; 57: 3=19)$ разница приращений $224-200=24 \ldots 3249^{+232} 3481$ $(\sqrt{3481}=59)^{+240} 3721(\sqrt{3721}=61) 3721^{+248} 3969(\sqrt{3969}=63 \in \in \mathrm{S} \ll 3 » \in \mathrm{S} \ll 33 »)$ Разница приращений: $248-224=24^{+256} 4225 \quad 4489(\sqrt{4489}=67)^{+272} \quad 4761(\sqrt{4761}=$ $69 \in \mathrm{S} « 3 »)$ Разница приращений: $272-248=24)^{+280} 5041(\sqrt{5041}=71)^{+288}$ $5329(\sqrt{5329}=73)^{+296}-5625(\sqrt{5625}=75)^{+304} 5929(\sqrt{5929}=77 \in Z)^{\in \mathrm{A}+312}$ $6241(\sqrt{6241}=79)^{+320} 6561\left(\sqrt{6561}=81=9^{2}=27 \times 3 ; \in S \ll 3 »\right) \ldots$

Зная приращение до ближайшего (наименьшего) квадрата сложного нечетного числа,-от «49» до «81»,- «32»,- можно определить все приращения квадратов сложных нечетных чисел и узнать значения этих чисел (для последующего извлечения корней квадратных и исключения их значений из ряда определяемых простых чисел). Приращение квадратов сложных от «81» равны: 32; 56; 80; 104; $128 ; 152 ; 176 ; 200 ; 224 ; 248 ; 272 ; 296 ; 320 ; 344 ; 368 ; 392 ; 416 ; 440 ; 464 ; 488 \ldots$ и далее до бесконечности по формуле: $\underline{\Delta S^{2}=32+24 x X} ; n=0 \rightarrow 81=9^{2}=49+32=7^{2}+32 \ldots n=1 \rightarrow 225$.

Выводы: 
1. n-порядки квадратов сложных нечетных чисел, четные и нечетные, в функции $\mathbf{Q}=\mathbf{9 + 8 n}$, цельноделимые на «3»,- подтверждают, что в значениях $\mathbf{Q}$ образованы квадраты сложных, делимых на «3». Числа этих порядков при определении простых,исключаются...(n=90 $\left.\rightarrow 729=27^{2} ; 27: 3=9 ; \mathrm{n}=324 \rightarrow 2601=51^{2} ; 51: 3=17\right) ; \mathrm{Q}=\mathrm{S}^{2}$, при n:3=X - целое,- исключаются...

2.Исключаются числа А-группы (делимые на «5», как сложные)... Исключаются $Z^{2}$ числа, образованные из гомогенной Z группы...

3.Исключаются сложные нечетные, как корни квадратные, определенные по формуле роста приращений квадратов сложных чисел, от 81: $\Delta \mathrm{S}^{2}=32+24 \mathrm{x}$, где

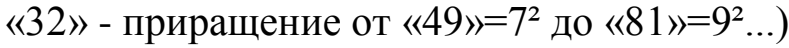

4.Исключаются промежуточные сложные (не квадраты) группы $\mathrm{S}$ «7» (119; 91 и другие (сами не квадраты, но образуют квадраты сложных).

5.Исключаются числа (возможно из других групп), образованные как корни квадратные из квадратов больших порядков; и значений в интервалах, образуемых функцией $\Delta \mathbf{S}^{2}=\mathbf{3 2}+\mathbf{2 4 x} \mathrm{X}, \mathbf{X} \neq \mathbf{0}, \mathbf{X} \rightarrow \infty \ldots$ Идентфикация производится использованием функций $\mathrm{Y}=5+6 \mathrm{xn},(\mathrm{n} \rightarrow \infty)$ и $\mathrm{Y}=5+7 \mathrm{xn}(\mathrm{n} \rightarrow \infty) \ldots$. Вероятно, простые числа, как промежуточные в функции $\mathrm{Y}=9+8 \mathrm{xn}(\mathrm{n} \rightarrow \infty),-$ должны в $\operatorname{Pr}$ «5» и $\quad \operatorname{Pr} « 7 »$ образовывать целые $\mathrm{n}$ - порядки...проверим это: $91=7+6 x n ; n=14 ; 91=5+6 x n ; n=14,3333 \ldots ; 119=7+6 x n ; n=18,6666 \ldots ; 119=5+6 x n$; $n=114: 6=19 ; 5+6 x n=35 ; n=5 ; 7+6 x n=35 ; n=4,6666 \ldots$ Видно, что ни одно промежуточное значение $\sqrt{\mathrm{X}^{2}}=\sqrt{9+8 n}$ (при определенных «n»), - не образовало целые n - порядки в обеих функциях...Промежуточные сложные, как 91; 119; исключаются как $\mathrm{Y}=\sqrt{9+8 \mathrm{xn}}$, при 9+8xn=91; 119 $\rightarrow \mathrm{n}=1034 ; 1769 \ldots$ Учитывая, что промежуточные (между квадратами сложных) приращения квадратов не четных чисел увеличиваются в каждом значении на «+8» и известны значения приращений квадратов сложных не четных чисел: $\Delta S^{2}=32+24 x X$, - можно узнать значения промежуточных приращений, обозначим как $\Delta \mathbf{P r}$... Рассмотрим интервалы [32; 56] ; [56; 80]. В первом интервале образуются $\Delta \operatorname{Pr}: 32+8=40 ; 40+8=48$;

$\Delta \mathrm{Pr}=40 \rightarrow 81+40=121=11^{2} ; 11 \in \operatorname{Pr} ; \Delta \operatorname{Pr}=48 \rightarrow 121+48=169=13^{2} ; 13 \in \operatorname{Pr} ; 169+56=225=15^{2} \in$ А; во втором интервале: $\Delta \mathrm{Pr}=56+8=64 ; \rightarrow 225+64=289=17^{2} ; 17 \in \operatorname{Pr}$; $\Delta \mathrm{Pr}=64+8=72 \rightarrow 289+72=361=19^{2} ; \quad 19 \in \mathrm{Pr}$. Тогда верно: $\operatorname{Pr}_{1}{ }_{1}=\mathrm{S}^{2}+\Delta \operatorname{Pr}^{2}{ }_{1} ; \operatorname{Pr}^{2}{ }_{2}=$

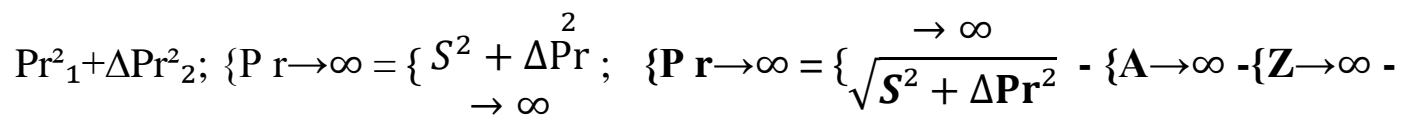
$\left\{\sqrt{S^{2}[\downarrow]} \rightarrow \infty\right.$ (промежуточные сложные, $\sqrt{S^{2}[\downarrow]}=\sqrt{9+8 n} \nleftarrow\left\{\begin{array}{l}\operatorname{Pr} " 5 " \\ \operatorname{Pr} " 7 "\end{array}\right.$

Обозначения: $[\downarrow]$ - означает значения внутри интервалов; $\{\rightarrow \infty$ - множество, 
стремящееся до бесконечности; $\nleftarrow\left\{\begin{array}{l}\operatorname{Pr} " 5 " \\ \operatorname{Pr} " 7 "\end{array}\right.$ квадратные, из значений внутри интервалов, - не образуются совместно в функциях Pr «5»=5+6xn и $\operatorname{Pr} « 7 »=7+6 x n,-$ в какой - либо функции образуется $\mathrm{n}$ - порядок с остатком; как корни квадратные промежуточные сложные определяются в $\mathrm{Y}=9+8 \mathrm{xn}$ при больших порядках... От «81» квадрат сложного числа можно определить так: $\mathbf{Q}=\mathbf{8 1}+(32+24 \times \mathbf{X})+\sum \Delta S^{2}[\downarrow]$, где $\sum \Delta S^{2}[\downarrow]$ - сумма приращений промежуточных квадратов нечетных, $S^{2}$ - предполагаемый квадрат простого числа (но, вероятно, $\mathbf{S}$ - сложные возможны)... X=1 $\rightarrow 81+(32+24)+40+48=225 ; \sqrt{225}=15 ; \mathrm{A} \leftarrow$ $15 \rightarrow S " 3 "$.

$\mathrm{X}=2 \rightarrow 81+40+48+56+64+72+80=441 ;(\sqrt{441}=21 \in \mathrm{S} « 3 " . \ll 80 »$ образуется как $32+24 \times 2=80$. Находим промежуточные числа между $\Delta \ll 81 »=32$ и 80,- (+8) -40; 48; 56; 64» 72; суммируем. Промежуточные сложные, как 91; 119; $\in \mathrm{S} « 7 " ;$ и другие, образованные при меньших $\mathrm{n}$ - порядках в функции $\mathrm{Y}=9+8 \mathrm{xn},-$ при приращениях, которые по предположению, должны были образовать простые числа, исключаются как $\sqrt{\boldsymbol{S}^{2}[\downarrow]}$ (промежуточные сложные), при появлении их при больших $\mathrm{n}-$ порядках в функции $\mathrm{Y}=9+8 \mathrm{xn}$, как образующих квадраты сложных чисел, образующихся при приращениях $\Delta \mathrm{S}^{2}=32+24 \mathrm{x} \mathrm{X}$, что отвечает интервалам приращений: [32; 56; ]; [56; 80; ]; [80; 104; ]; [104; 128; ]; [128; 152; ]; [152; 176; ] и далее, до $\infty$, с ростом «+24хX.». Значит, простые числа - значения, если $P \mathbf{r}=\sqrt{\mathbf{S}^{2}+\Delta \mathbf{P r}^{2}} \rightarrow X, \ldots$ (с остатком; с исключением $\mathrm{A} ; \mathrm{Z}$; и $\boldsymbol{S}[\downarrow]$ (промежуточных сложных). Если существует предполагаемая закономерность образования $\mathrm{n}$ - порядков простых чисел в функции $\quad \operatorname{Pr}=9+8 x n$, изложено ранее), - приращение $\Delta \mathrm{n}-$ порядка при каждом значении числа увеличивается на $«+1 »(2 \stackrel{+3}{\rightarrow} 5 ; 5 \stackrel{+4}{\rightarrow} ; 9 \stackrel{+5}{\rightarrow} ; 14 \stackrel{+6}{\rightarrow} ; 20 \stackrel{+7}{\rightarrow} ; 27 \stackrel{+8}{\rightarrow}$; $35 \stackrel{+9}{\rightarrow} ; 44 \stackrel{+10}{\longrightarrow} ; 54 \stackrel{+11}{\longrightarrow} ; 65 \stackrel{+12}{\longrightarrow} ; 77 \stackrel{+13}{\longrightarrow} ; 90 \stackrel{+14}{\longrightarrow} ; 104 \stackrel{+15}{\longrightarrow} ; 119 \stackrel{+16}{\longrightarrow} ; 135 \stackrel{+17}{\longrightarrow} ; 152 \stackrel{+18}{\longrightarrow} ; 170 \stackrel{+19}{\longrightarrow}$; $189 \stackrel{+20}{\longrightarrow} 209,-$ можно построить график квадратов (их корней) простых чисел до бесконечности. Рассмотрим образование простых чисел в функции $\operatorname{Pr}=\sqrt{9+8 n}(\mathrm{n}-$ порядок числа) 


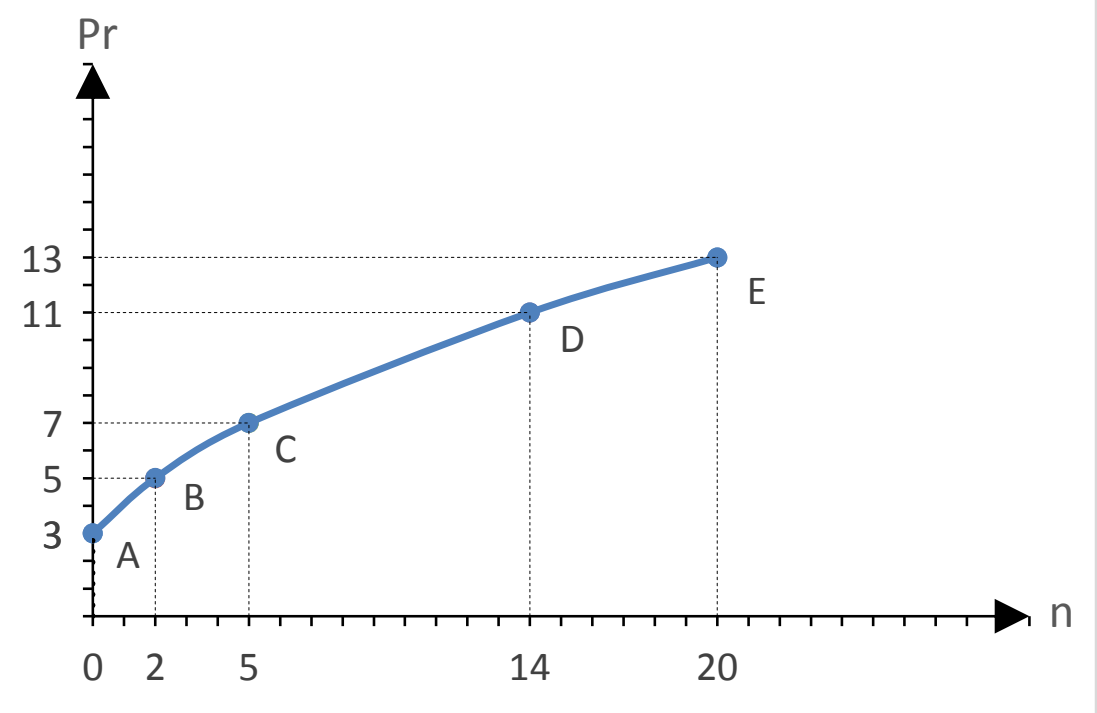

Рис.1. Зависимость значений простых чисел от их $\mathrm{n}$ - порядков

Образуется параболическая кривая зависимости значений простых чисел от их $\mathrm{n}-$ порядков функции $\operatorname{Pr}=\sqrt{9+8 n} \mathrm{n}=0 \rightarrow 3 ; \mathrm{A}(0 ; 3) ; \mathrm{n}=2 \rightarrow 5 ; \mathrm{B}(2 ; 5) . \mathrm{n}=5 \rightarrow 7 ; \mathrm{C}(5 ; 7)$; $\mathrm{n}=14 \rightarrow 11 ; \mathrm{D}=20 \rightarrow 13 ; \mathrm{E}(20 ; 13)$.

Если считать простые числа корнями квадратными из их квадратов, образуются точки графика $\mathrm{Y}=\mathrm{Pr}^{2}$. Точки графика : A(3;9); В(5;25); C(7;49); D(11;121); E(13;169). Образуется мало искривленная линия, под наклоном к оси $\mathrm{X}(\mathrm{Pr})$, близким к вертикальному, как предполагал Риман...

Итак, $\left\{\operatorname{Pr}^{a} \sqrt{S^{2}+\Delta} \operatorname{Pr}^{2}(=X-\right.$ нечетное число с остатком, при целых $\mathbf{n}$ - порядках $\operatorname{Pr}^{2}($ в $Y=9+8 x n$ (a - четное значение; стремится от $\infty$ ко второй степени для определения простого числа. $\{\boldsymbol{a}(\infty \rightarrow 2)$.

Построим график функции $\mathrm{Y}=\operatorname{Pr}^{2}(\mathrm{X})$. C увеличением значений «X» наклон к оси абсцисс уменьшается, кривая приобретает вид вертикальной прямой. Именно вертикальная прямая, как предполагал Риман, показывает образование простых чисел из их квадратов. 


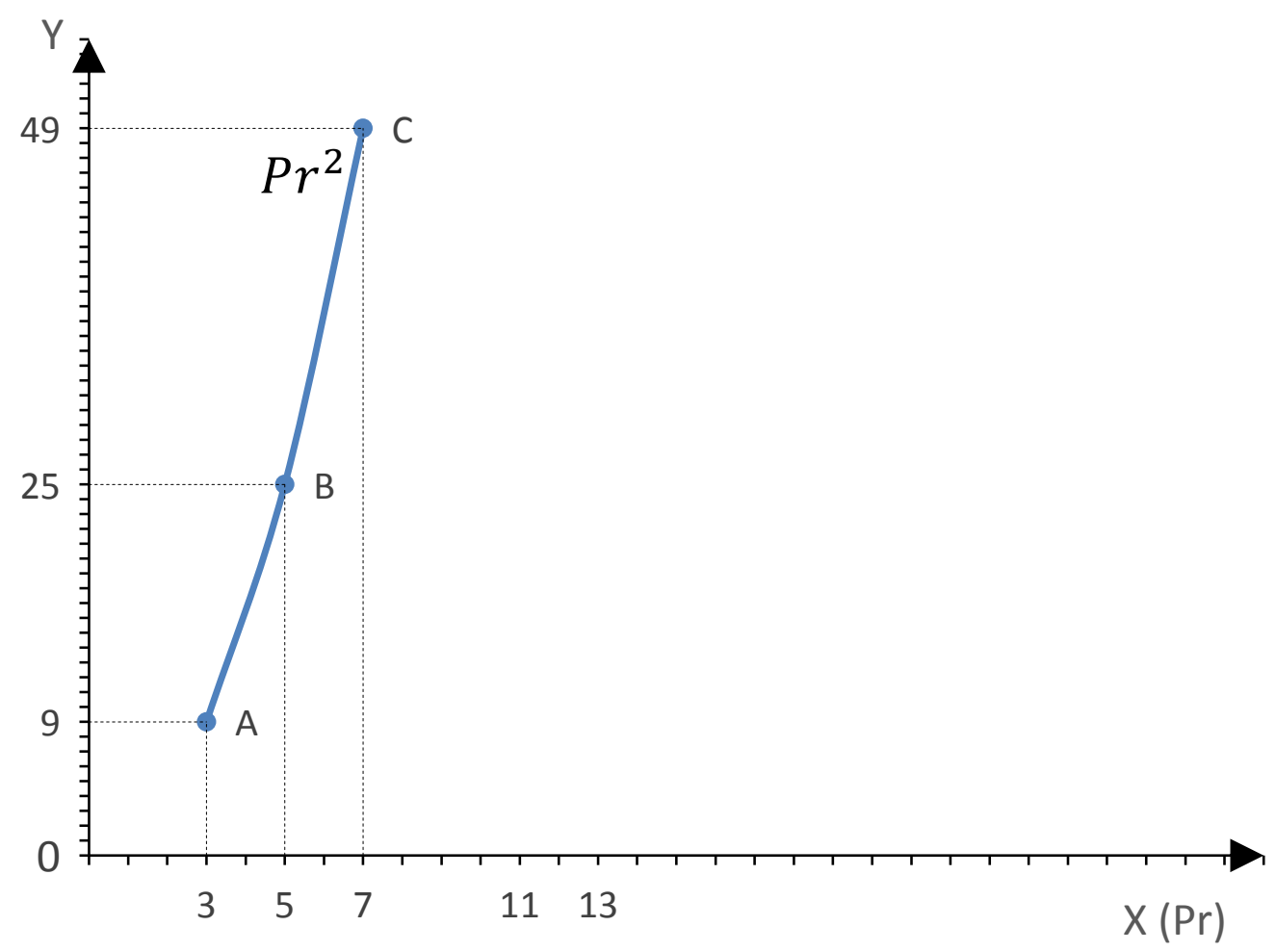

Рис.2. График функции $\mathrm{Y}=\operatorname{Pr}^{2}(\mathrm{X})$

В Y $=\sqrt{9+8 \mathrm{xn}}$ образуются и простые, и сложные нечетные числа...

$\mathrm{n}=27 \rightarrow \sqrt{225}=15 ; \mathrm{n}=35 \rightarrow \sqrt{289}=17 ; \mathrm{n}=44 \rightarrow \sqrt{361}=19 ; \mathrm{n}=54 \rightarrow \sqrt{441}=21$;

$\mathrm{n}=65 \rightarrow \sqrt{529}=23 ; \mathrm{n}=77 \rightarrow \sqrt{625}=25 ; \mathrm{n}=90 \rightarrow \sqrt{729}=27 ; \mathrm{n}=104 \rightarrow \sqrt{841}=29 \ldots$ д далее, до $\infty \ldots$

Задача произвести идентификацию простых чисел... С другой стороны известно, как от $81=9^{2}$ (квадрат первого сложного нечетного числа) - определять квадраты сложных нечетных ...Формула квадратов сложных нечетных чисел: $\mathrm{Q}=\mathbf{8 1}+(32+24 \mathrm{XX})++\sum \Delta \boldsymbol{S}^{2}[\downarrow]$, где $\sum \Delta \boldsymbol{S}^{2}[\downarrow]$ - сумма приращений промежуточных квадратов нечетных чисел (квадратов простых, $\mathrm{Pr}^{2}$ ), -отображено выше.

Учитывая это, все квадраты сложных нечетных чисел для формирования группы квадратов простых нечетных чисел следует исключить.

Таким образом, чтобы получить простые числа, следует из множества чисел функции $\mathrm{Y}=\sqrt{9+8 \mathrm{xn}}$, где способ определения $\mathrm{n}$ - порядков (по таблице, способ ее составления указан выше), -нужно исключить множество квадратов сложных нечетных чисел начиная от «81». Если множество обозначается как $\{\rightarrow \infty$, то 
множество Рг определяется так: $\{\mathbf{P} \mathbf{r} \rightarrow \infty=\{\rightarrow \infty \sqrt{\mathbf{9 + 8 \times n}}-\{\rightarrow \mathbf{8 1}+(\mathbf{3 2}+\mathbf{2 4 x} \mathbf{X})+$ $\sum \Delta \boldsymbol{S}^{2}[\downarrow]$. (Подчеркнуто единое множество).

Следует исключить также значения корней квадратных (извлеченные из $\left\{\rightarrow 81+(32+24 \mathrm{xX})+\sum \Delta S^{2}[\downarrow]\right.$ и сложные нечетные, образуемые в $\mathrm{Y}=\sqrt{9+8 \mathrm{xn}}$

До «81», - $15 ; 21 ; 25 ; 27 ; 33 ; 35 ; 39 ; 45 ; 49 ; 51 ; 55 ; 57 ; 63 ; 65 ; 69 ; 75 ; 77$. Группу этих сложных обозначим как интервал [15 ${ }^{\leftarrow S \rightarrow 77]}$ (S - сложные).

Тогда верно: (множество простых чисел до бесконечности определяется:

$\left\{\mathbf{P} \mathbf{r} \rightarrow \infty=\left\{\sqrt{\mathbf{9}+\mathbf{8} \times \mathbf{n}, \rightarrow \infty}-\left\{\rightarrow \mathbf{8 1}+(\mathbf{3 2}+\mathbf{2 4 x X})+\sum \Delta S^{2}[\downarrow], \rightarrow \infty-\right.\right.\right.$

$-\left\{\sqrt{\rightarrow \mathbf{8 1}+(32+24 \mathrm{xX})+\sum \Delta S^{2}[\downarrow], \rightarrow \infty}-\left[15^{\leftarrow S \rightarrow 77}\right]\right.$.

Образование $\mathrm{n}$ - значений рассмотрено ранее, по схеме: $2 \stackrel{+3}{\rightarrow} 5 ; 5 \stackrel{+4}{\rightarrow} ; 9 \stackrel{+5}{\rightarrow} ; 14 \stackrel{+6}{\rightarrow} ; 20 \stackrel{+7}{\rightarrow}$; $27 \stackrel{+8}{\rightarrow} ; 35^{+9} \rightarrow \ldots$, по формуле $\{\mathbf{n} \rightarrow \infty=\mathbf{2}+\{\Delta \mathbf{n} «+\mathbf{1} \gg \rightarrow \infty$ в каждом следующем $\mathbf{n}-$ значении. $\{\Delta \mathrm{n}$ «+ $1 » \rightarrow \infty,-$ множество приращений $\mathbf{n}-$ значений (от «2», при увеличении каждого $\Delta \mathbf{n}-$ последующего на «+1» $\sum \Delta S^{2}[\downarrow]$ сумма приращений промежуточных квадратов нечетных чисел, квадратов простых чисел.(рассмотрено ранее)...

$\left\{\sqrt{\rightarrow \mathbf{8 1}+(32+24 \mathrm{XX})+\sum \Delta \boldsymbol{S}^{2}[\downarrow], \rightarrow \infty},-\right.$ множество целых чисел, определяемое для получения и исключения минимальных значений квадратов сложных чисел. По факту значения $\left\{\rightarrow 81+(32+24 \mathrm{xX})+\sum \Delta S^{2}[\downarrow], \rightarrow \infty\right.$ и $\sqrt{\rightarrow 81+(32+24 \mathrm{xX})+\sum \Delta S^{2}[\downarrow], \rightarrow \infty}$ - должны совпадать, быть одинаковыми.

Ранее был сделан вывод, что промежуточные (между квадратами сложных нечетных), приращения квадратов нечетных (предполагаемых простых), увеличиваются в каждом значении на «+8», и известны приращения квадратов сложных нечетных чисел, что определяются по формуле: $\Delta \mathrm{S}^{2}=32+24 \mathrm{XX}$ (от «81=92»). Значит, можно узнать значения приращений ожидаемых квадратов простых, $\Delta \operatorname{Pr}^{2}$.

Рассмотрим интервалы при различных «X-значениях»: [32; 56; ]; [56; 80; ]...в первом образуются , $\Delta \mathrm{Pr}^{2}: 32+8=40 ; 40+8=48 ;, \Delta \mathrm{Pr}^{2} .=40 \rightarrow 81+40=121=11^{2} ; 11 \in \mathrm{Pr}$, $\Delta \mathrm{Pr}^{2}=48 \rightarrow 121+48=169=13^{2} ; 13 \in \operatorname{Pr}$. Во втором интервале образуются:

$\Delta \operatorname{Pr}^{2}=56+8=64 \rightarrow 225+64=289=17^{2} ; 17 \in \operatorname{Pr}$. (Примечание: крайним значениям интервалов в зависимости $\Delta \mathbf{S}^{2}=\mathbf{3 2}+\mathbf{2 4 x} \mathbf{X}$, - соответствуют квадраты сложных нечетных чисел, как $56 \rightarrow 225=15^{2}(169+56=225) \ldots \Delta \operatorname{Pr}^{2}=64+8=72 \rightarrow 289+72=361=19^{2}$; $19 \in \operatorname{Pr} .$. 
То есть зная, что при $\mathrm{X}=0, \Delta \mathrm{S}^{2}=32$ образуется «81=92»), можно рассчитать все квадраты сложных $\left(\operatorname{Pr}^{2}\right)$ до бесконечности. Логично что $\mathbf{P r}^{2}=\mathbf{S}^{\mathbf{2}}+\Delta \mathbf{P r}^{\mathbf{2}}$ в заданном интервале $\left[\Delta \mathbf{S}_{\mathbf{1}}{ }^{2} ; \Delta \mathbf{S}^{2}\right]$

Примечание! В формуле выше в значении $\sum \Delta \boldsymbol{S}^{2}[\downarrow]$ - сумма приращений квадратов простых чисел использован символ $\mathbf{S}$, как показывающий, что сумма приращений квадратов простых чисел с позади стоящим квадратом сложного числа, -образует квадрат сложного числа в крайних значениях интервалов. К примеру:

$81+40+48+56=225=15^{2} ; 40+48=96-$ есть значение $\sum \Delta \boldsymbol{S}^{2}[\downarrow]$.

Зная приращения квадратов простых чисел и квадраты простых, им соответствующие, можно построить график зависимости $\operatorname{Pr}^{2} \rightleftarrows \Delta \operatorname{Pr}^{2}$

Возьмем минимальные значения $\Delta \operatorname{Pr}^{2}: 40 ; 48 ; 64 ; 72$. Им соответствуют значения $\operatorname{Pr}^{2}: 121 ; 169 ; 289 ; 361$.

Видно, что кривая зависимости $\operatorname{Pr}^{2} \rightleftarrows \Delta \operatorname{Pr}^{2}$ постепенно, с увеличением $\Delta \operatorname{Pr}^{2}$ выпрямляется вертикально к оси абсцисс « $\Delta \operatorname{Pr}^{2} »$, что предполагал Риман.



Рис.3. Кривая зависимости $\operatorname{Pr}^{2} \rightleftarrows \Delta \operatorname{Pr}^{2}$ 
Как определять значения простых чисел до бесконечности, показывают выведенные формулы выше... Можно сделать общие выводы о простых числах.

1. $\left\{\mathrm{P} \mathrm{r} \rightarrow \infty=\mathrm{Q}\right.$ (все нечетные) - $\left\{\mathrm{P} \mathrm{r}^{\mathrm{n}} \rightarrow \infty\right.$, где $\mathrm{n}$ - четная степень простых чисел, начиная от квадратной (второй) $-\{\mathrm{S}[\downarrow] \rightarrow \infty$ (множество промежуточных сложных нечетных чисел, не образованных четной степенью числа.

$2 \cdot \sqrt[n]{\{\mathrm{S}[\downarrow]} \rightarrow \infty=\mathrm{X}, \ldots-$ из множества промежуточных сложных чисел при $\mathrm{n}-$ четной степени получаются числа с остатком.

3. $\sqrt[n]{\{\mathrm{S}[\downarrow]} \rightarrow \infty=\mathrm{X}$ - целые значения корней из промежуточных сложных могут образовываться при нечетных $\mathrm{n}-$ значениях, к прмеру: $\sqrt[3]{27}=3 ; \sqrt[3]{343}=7 ; \sqrt[3]{243}=3$.

\section{4. Промежуточные сложные числа (не четные), образуются произведением} простых чисел: $\left\{S[\underline{\downarrow}] \rightarrow \infty=\left\{\mathbf{P} \mathbf{r}_{1} x \mathbf{P} \mathbf{r}_{2} \rightarrow \infty\right.\right.$,при этом $\mathbf{P} \mathbf{r}_{1} \neq \mathbf{P} \mathbf{r}_{2}$ (простые числа разные).

К примеру: $11 \times 13=143 ; 11 \times 17=187 ; 3 \times 73=219 ; 17 \times 67=1139$. Соответственно, общий вывод:

1.Следует дополнить, что промежуточные сложные, $\{\mathrm{S}[\downarrow] \rightarrow \infty \infty,-$ это не только значения нечетных степеней простых чисел (как 27), но и числа, образованные произведением простых...

Это можно отобразить так:

\section{1. $\left\{\mathbf{P} \mathbf{r}_{1} \times \mathbf{P} \mathbf{r}_{2} \rightarrow \infty\left(\mathbf{P} \mathbf{r}_{1} \neq \mathbf{P} \mathbf{r}_{2}\right)\right.$}

\section{2. $\left\{\mathbf{P} \mathbf{r}^{\mathrm{n}} \rightarrow \infty, \mathbf{n}-\right.$ нечетное число $\in\{\mathbf{S}[\downarrow] \rightarrow \infty$}

1-е и 2-е множества исключаются из ряда Q всех нечетных при определении простых чисел.

Решение гипотезы Римана имеет значение для современных вычислительных, компьютерных технологий...

Решение ее позволяет определить все простые числа до бесконечности...

Рассмотрим связь предположений Римана с образованием простых чисел, их квадратов... Если Z - функция Римана функция, которая любому действительному числу $\mathbf{S}$ ставит в соответствие сумму значений ряда, $\mathbf{S}=\mathbf{6}+\mathbf{i t}, \mathbf{и л и} \mathbf{S}=\mathbf{a}+\mathbf{b} \mathbf{i}$, где i мнимое (предполагаемое число); б; $\mathrm{t}$ или a; b - действительные числа, то вероятно, что есть числа, образующие (участвующие в образовании) простые, их квадраты, квадраты сложных нечетных, - по принципу этих формул(лы), применяющемся 
значении it. Такие числа называются комплексными, образующимися функцией комплексного переменного.С - комплексное число, имеет действительную и мнимую часть... Модуль (абсолютное значение) комплексного числа равен $\mathrm{Z}=\sqrt{a^{2}+b^{2}} \ldots$ Ранее указывалось, что для определения простых чисел до бесконечности надо вычислить квадраты простых чисел (до бесконечности), зная приращения квадратов простых «если простые числа считать комплексными, то их модуль равен: $\mid\left\{\boldsymbol{P r} \rightarrow \infty|=|\left\{\sqrt{\boldsymbol{S}^{2}+\Delta \boldsymbol{P r}^{2}} \mid\right.\right.$, где $\boldsymbol{S}^{2}$ - квадрат сложного нечетного числа; $\Delta P r^{2}$ - приращение квадрата простого, впереди стоящего числа. Расчет $\Delta P r^{2}$ начинаем от «32» (с увеличением каждого впереди стоящего приращения квадрата на «8»). Можно составить таблицу приращений квадратов простых чисел: 32+8=40; 40+8=48; 56; 64; 72; 80; 88; 96; 104,...и далее до бесконечности, с увеличением на «+8». Видно, что модуль комплексного числа и простые числа, исходя из возможности определять их квадраты до бесконечности, определяются принципиально одинаково. Тогда любое простое число можно считать комплексным, состоящим из двух действительных чисел, суммируемых между собой и являющихся квадратом сложного и квадратом приращения простого. Из их суммы извлекается корень второй степени... Вероятно, приращение квадрата простого можно представить как комплексное число, имеющее мнимую часть, что и дает предположение о комплексности простых чисел... Сложные нечетные числа определяются как корни квадратные из их квадратов, начиная от $81=9^{2}(9-$ первое сложное в ряду нечетных Q)... Зная, как образуются приращения квадратов сложных:

$\Delta S^{2}=32+24 \times X(32$ соответствует 81, квадрату первого сложного нечетного, 9), можно узнать значения квадратов всех сложных нечетных до бесконечности). Для этого надо суммировать приращения квадратов нечетных (в том числе и простых) до (включительно) приращения квадрата искомого сложного нечетного числа, соответствующего определенному значению $\mathbf{X}$ в формуле $\Delta \mathbf{S}^{\mathbf{2}}=\mathbf{3 2}+\mathbf{2 4} \mathbf{x} X$. При $\mathrm{X}=0 \rightarrow 81$ (первый квадрат минимального сложного,9)... Возьмем $\mathrm{X}=5$, образуется: $32+24 \times 5=152 \ldots$ Промежуточные приращения квадратов: $32+8=40 ; 48 ; 56 ; 64 ; 72 ; 80$; $88 ; 96 ; 104 ; 112 ; 120 ; 128 ; 136 ; 144 \ldots$ то есть: $S^{2}=81+\sum$ выше перечисленных чисел $+152=1521 ; \sqrt{1521}=39 ;(39: 3=13)$. Зная приращения квадратов простых и сложных нечетных чисел, можно составить таблицу приращений квадратов нечетных (сложных и простых до бесконечности, начиная от 32 $\rightarrow$ 81) . . Подчеркнуты

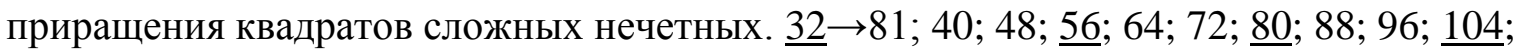
$112 ; 120 ; \underline{128} ; 136 ; 144 ; \underline{152} ; 160 ; 168 ; \underline{176} ; 184 ; 192 ; \underline{200} ; 208 ; 216 ; \underline{224} ; 232 ; 240 ; \underline{248}$; $256 ; 264 ; 272 ; 280 \ldots$ и далее до бесконечности. Видно, что любому квадрату сложного и простого числа соответствует сумма определенных значений приращений квадратов нечетных (назовем ряд Z), начиная (условно) с $32 \rightarrow 81=9^{2}$. Дзета - функция Римана об этом и говорит. Как найти нули дзета - функции? Дзета функция, - функция комплексного переменного... Говоря о ней, следует вспомнить о 
производной... Производная, - основное понятие дифференциального исчисления , характеризует скорость изменения функции... Определяется как предел отношения прироста функции к приросту ее аргумента при стремлении прироста аргумента к «О», если такой предел существует...их образование бесконечно... Производная равна: $\oint^{\prime}(\mathrm{X})=\frac{\Delta \oint}{\Delta \mathrm{X}}$,

Дзета - функция Римана является аналитической и функция зависимости приращений квадратов (простых и сложных), должна быть у них тождественна, то есть выполняется условие Коши - Римана: $\frac{\Delta \mathrm{Y}_{1}}{\Delta \mathrm{X}_{1} r}=\frac{\Delta \mathrm{Y}_{2}}{\Delta \mathrm{X}_{2} r} ;: \frac{\Delta \mathrm{Y}_{1}}{\Delta \mathrm{X}_{2} r}=-\frac{\Delta \mathrm{Y}_{2}}{\Delta \mathrm{X}_{1} r}$, где $\Delta \mathrm{Y}_{1}$ приращения (разница между квадратами сложных нечетных $\Delta \mathrm{X}_{1} r$ - разница между приращениями квадратов сложных нечетных. $\Delta \mathrm{Y}_{2}$ - разница между квадратами простых. $\Delta \mathrm{X}_{2} r$ - разница между приращениями квадратов простых... Проверим это равенство...

$32 \rightarrow 81 ; 56 \rightarrow 225$ (для сложных); 40 $\rightarrow 121 ; 48 \rightarrow 169$ (для простых); 225-81=144; 56$32=24 ; \Delta \mathrm{Y}_{1}=144 ; \Delta \mathrm{X}_{1}=24 ; 144: 24=6 ; 169-121=48 ; 48-40=8 ; 48: 8=6 ;$ возьмем $21^{2}=441 ; \rightarrow \Delta \mathrm{X}_{1}=80 ; \Delta \mathrm{X}_{2}=72 \rightarrow 361=19^{2} ; 441-225=216 ; 80-56=24 ; 216: 24=9$. Разницу между квадратами сложных и их приращениями, результат деления определили. Теперь то же сделаем с квадратами простых, расположенных между 225 и 441, то есть $289=17^{2}$ и $361=19^{2} .72-64=8\left(\Delta \mathrm{X}_{2} r-\right.$ разница между приращениями квадратов простых, $\Delta \mathrm{X}_{2}=72$ и $\Delta \mathrm{X}_{2}=64$, -равна 8). $361-289=72 ; 72: 8=9$. Такой же результат деления, 9, образуется и при рассмотрении крайних значений интервала [225; 289 ;

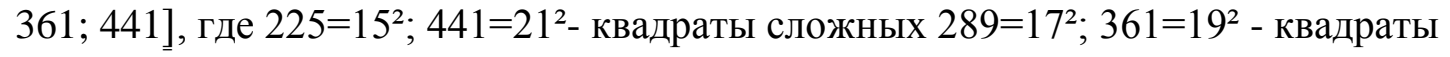
простых чисел.

Вывод: в бесконечном ряду квадратов нечетных чисел (сложных и простых) наблюдается закономерность - внутри интервалов с двумя крайними значениями квадратами сложных и двумя между, квадратами простых, - соотношения разницы квадратов к разнице соответствующих им приращений равны, соотношения для сложных равны соотношениям для простых, то есть выполняется условие Коши Римана.

$\frac{\Delta \mathrm{Y}_{1}}{\Delta \mathrm{X}_{1} r}-\frac{\Delta \mathrm{Y}_{2}}{\Delta \mathrm{X}_{2} r}=\mathbf{0}$

Этим равенством объясняется расположение «нулей дзета - функции Римана». Они образуются согласно данному условию в интервалах связи роста значений квадратов простых и сложных от их приращений, их разницы. $\left[32 \rightarrow 81=9^{2}\right.$; $\left.40 \rightarrow 121=11^{2} ; 48 \rightarrow 169=13^{2} ; 56 \rightarrow 225=15^{2}\right],\left[56 \rightarrow 225=15^{2} ; 64 \rightarrow 289=17^{2} ; 72 \rightarrow 361=19^{2} ;\right.$ $\left.80 \rightarrow \mathbf{4 4 1}=\mathbf{2 1 ^ { 2 }}\right]$, аналогично, до бесконечности. Если значениями аргумента считать значения $\mathrm{X}=\frac{\Delta \mathrm{Y}_{1}}{\Delta \mathrm{X}_{1} r}-\frac{\Delta \mathrm{Y}_{2}}{\Delta \mathrm{X} 2 r}$ внутри интервалов связи, то на оси абсцисс все значения $\mathbf{X}$ будут соответствовать «0». На оси Y приращениям будут соответствовать квадраты 
нечетных чисел (начинаем от $81=9^{2}$ согласно предыдущим расчетам. Данную функцию Z следует считать комплексной, выразив совокупностью:

$$
f(Z)=\left\{\begin{array}{l}
\mathrm{X}=\frac{\Delta Y 1}{\Delta X 1 r}-\frac{\Delta Y 2}{\Delta X 2 r} ; \\
\mathrm{Y}=\text { от } 81 \rightarrow \infty ; \mathrm{S}^{2} ; \mathrm{Pr}^{2} .
\end{array}\right.
$$

Значение $81=9^{2}$ можно считать константой, постоянной величиной от которой происходит рост квадратов (сложных и простых)...

Видно, что образуется вертикальная прямая, соответствующая оси Ү,как предполагал Б.Риман.

Значению $81=9^{2}$ соответствует $\Delta \mathrm{X}_{1}=32 \ldots$ Величину «32», от которой узнаем приращения, следует считать const, от которой происходит рост по принципу комплексного числа: $\mathrm{Z}=\mathrm{a}+\mathrm{bi}$, где $\mathrm{a}-$ действительная часть, соответствует const; $\mathrm{b}$ мнимая часть, соответствует приращению квадрата нечетного числа. $32+8=40 \stackrel{\text { образует }}{\longrightarrow}$ $81+40=121=11^{2} ; 11 \in \operatorname{Pr} .32+8+8=48 \stackrel{\text { образует }}{\longrightarrow} 81+40+48=169=13^{2} ; 13 \in \operatorname{Pr} .32+8+8+8=56$ образует $\stackrel{\text { образует }}{\longrightarrow} 81+40+48+56=225=15^{2} ; 15 \in$ А.(сложное A - группы). Мнимая часть (предполагаемая ) - величина не постоянная, в качестве приращения зависит от величины значений квадратов нечетных чисел, то есть переменная, образуемая комплексом (суммой) приращений от 32 на $+8 \mathrm{xn}-$ значений. Значение «8xn» есть мнимая часть, от него зависит (от n), значение функции комплексной переменной (величина приращения).

Среднее значение суммы приращений в интервалах [32; 56]; [56; 80]; [80; 104]; [104; 128] и далее до бесконечности, (сумма крайних значений, для сложных), не равно приращениям квадратов простых во всех интервалах, приращения квадратов простых отклоняются от середины. В интервалах $\left[\Delta \mathbf{S}_{1}{ }^{2} ; \Delta \operatorname{Pr}_{1}{ }^{2} ; \Delta \operatorname{Pr}_{2}{ }^{2} ; \Delta \mathbf{S}_{2}{ }^{2}\right]$ это выражается так: $\frac{\Delta \mathrm{S}_{1}{ }^{2}+\Delta \mathrm{S}_{2}{ }^{2}}{2} \neq \Delta \operatorname{Pr}_{1}{ }^{2} ; \Delta \operatorname{Pr}_{2}{ }^{2}$ (отсутствие равенства, о чем утверждал Риман)

Примеры: 32+56=88; 88:2=44. Приращения квадратов простых внутри данного интервала 40; 48. В интервале [56; 80] среднее значение $(56+80): 2=68$; приращение квадратов простых 64; 72. В [104; 128] среднее значение 116; приращения квадратов простых 112; 120. В [128;152] среднее значение 140; приращения $\operatorname{Pr}_{1}^{2} ; \operatorname{Pr}_{2}^{2}$ равны $136 ; 144 ; \mathrm{B}[152 ; 176]$ среднее значение $164 ; \Delta \operatorname{Pr}_{1}{ }^{2}=160 ; \Delta \operatorname{Pr}_{2}{ }^{2}=168$. Видно, что приращения квадратов простых чисел в интервалах связи квадратов (их 
приращений) нечетных чисел, - отличаются от среднего арифметического значения суммы крайних значений на $\mp 4$. Эту зависимость назовем равноудаленностью приращений квадратов простых чисел. $\Delta \operatorname{Pr}_{1}{ }^{2}=\frac{\Delta S_{1}{ }^{2}+\Delta S_{2}{ }^{2}}{2}-4 . \Delta \operatorname{Pr}_{2}{ }^{2}=\frac{\Delta S_{1}{ }^{2}+\Delta S_{2}{ }^{2}}{2}+4$. Равноудаленность приращений обеспечивает упорядоченное образование простых чисел...



Функция Римана, Z-функция комплексного переменного, где все нули - значения «Х» (условие Коши-Римана). $\mathrm{y}_{1} ; \mathrm{y}_{2}$ - приращения квадратов простых чисел, начиная от $121=11^{2}\left(\Delta \operatorname{Pr}^{2}=40\right)$. 
Значения $\left\{\mathrm{y}_{1} ; \mathrm{y}_{2} \rightarrow \infty\right\}=\{40 ; 48 ; 64 ; 72 ; 112 ; 120 ; 136 ; 144$, и далее, до бесконечности $\}$. Значениями $\Delta \operatorname{Pr}_{1}^{2}$ и $\Delta \operatorname{Pr}_{2}^{2}$ есть $\frac{1}{2}$ комплексного переменного, $\left(\Delta \mathrm{S}_{1}{ }^{2}+\Delta \mathrm{S}_{2}^{2}\right) \pm 4$, отклоняющегося от среднего значения на \pm 4 .

$$
f(Z)=\left\{\begin{array}{l}
\mathrm{X}=\frac{\Delta Y 1}{\Delta X 1 r}-\frac{\Delta Y 2}{\Delta X 2 r} ;(0) . \\
\mathrm{y}_{1}=\Delta \operatorname{Pr}_{1}^{2}=\frac{\Delta \mathrm{S} 12+\Delta \mathrm{S} 22}{2}-4 ;\left(\mathrm{S}_{1} \text { и } \mathrm{S}_{2} \text { в квадрате }\right) \\
\mathrm{y}_{2}=\Delta \operatorname{Pr}_{2}{ }^{2}=\frac{\Delta \mathrm{S} 12+\Delta \mathrm{S} 22}{2}+4 ;\left(\mathrm{S}_{1} \text { и } \mathrm{S}_{2} \text { в квадрате }\right)
\end{array}\right.
$$

Примечание: выражение функции Римана в виде

$$
f(Z)=\left\{\begin{array}{l}
\mathrm{X}=\frac{\Delta Y 1}{\Delta X 1 r}-\frac{\Delta Y 2}{\Delta X 2 r} ; \\
\mathrm{Y}=\left\{\text { от } 81 \rightarrow \infty ; \mathrm{S}^{2} ; \mathrm{Pr}^{2} .\right.
\end{array}\right.
$$

указывает на зависимость образования квадратов нечетных чисел от их приращений.

Учитывая, что все х-значения в Z-функции Римана, согласно выполнению условия КошиРимана, всегда равны «0», а приращения квадратов простых чисел отклоняются от среднего значения приращений квадратов сложных (в установленных интервалах) на \pm 4 , получим краткую формулу Z-функции Римана:

$f(Z)=\mathrm{X}+\Delta \operatorname{Pr}_{1}^{2} ; \Delta \operatorname{Pr}_{2}^{2}$ или, $(\mathrm{X}=0)$

$f(Z)=\frac{\Delta \mathrm{S} 12+\Delta \mathrm{S} 22}{2} \pm 4$ - функция полусуммы значений аргумента с отклонением для $\Delta \operatorname{Pr}_{1}^{2}$ на «- 4»; для $\Delta \operatorname{Pr}_{2}^{2}$ на «+4» от среднего, как предполагал Риман.

\section{References}

1. Vilenkin, Schwarzburd. "Matematicheskij analiz” ["Mathematical analysis"], 1969.

Translation of the Title, Abstract and References to the Author's Language

$$
\begin{gathered}
\text { Установление закономерности образования и } \\
\text { распределения простых чисел до бесконечности } \\
\text { (гипотеза Римана) }
\end{gathered}
$$




\section{Мустафаев Рустем Эйвасович}

\section{Аннотация}

В работе проанализировано образование простых чисел в связи со сложными нечетными числами, установлена формула для определения простых чисел до бесконечности.

Простые числа, расположение которых пытались объяснить с помощью асимптотического закона распределения простых чисел, важны в криптографии, навигации, имитационном моделировании, то есть их определение имеет практическое значение.

Ключевые слова: $\operatorname{Pr}$ (простое число); S - (сложное число); A - группа; S «5»; Z - группа; D - группа; образующий остаток; абсолютный вектор I $\overrightarrow{\mathrm{I}} \mathrm{I}$; основа; -; корень; корень квадратный; в функции Рr; подгруппа $\mathrm{S}$ «33»; множество X; квадрат нечетных; $\mathrm{n}$ - порядки; функция $\operatorname{Pr}$ «13»; квадраты сложных; приращение $(\Delta \mathrm{n}) ; \mathrm{Q}$ - значения; $\Delta \mathrm{n}=\mathrm{n}_{2}-\mathrm{n}_{1} ; \mathrm{n}$ - четная степень; объединяющее число; $\mathrm{S}[\downarrow]$ промежуточные сложные; идентификация; приращения квадратов; интервал; от «81»; сумма приращений; исключить; $\{\mathrm{P} \mathrm{r} \rightarrow \infty$.

Лuтература

1. Виленкин, Шварцбурд «Математический анализ», 1969. 